\title{
A MODIFIED PARTICLE METHOD FOR SEMILINEAR HYPERBOLIC SYSTEMS WITH OSCILLATORY SOLUTIONS*
}

\author{
R. C. FETECAU ${ }^{\dagger}$ AND T. Y. HOU ${ }^{\ddagger}$ \\ Dedicated to Prof. George Papanicolaou with friendship and admiration
}

\begin{abstract}
We introduce a modified particle method for semi-linear hyperbolic systems with highly oscillatory solutions. The main feature of this modified particle method is that we do not require different families of characteristics to meet at one point. In the modified particle method, we update the $i$ th component of the solution along its own characteristics, and interpolate the other components of the solution from their own characteristic points to the $i$ th characteristic point. We prove the convergence of the modified particle method essentially independent of the small scale for the variable coefficient Carleman model. The same result also applies to the non-resonant Broadwell model. Numerical evidence suggests that the modified particle method also converges essentially independent of the small scale for the original Broadwell model if a cubic spline interpolation is used.
\end{abstract}

Key words. Particle methods, homogenization, hyperbolic systems

AMS subject classifications. 65M12, 74Q10

1. Introduction. Particle methods have been demonstrated to be effective in approximating oscillatory solutions of semi-linear hyperbolic differential equations. In $[5,6,7,4]$, it was showed that by using a sampling technique and a particle method approximation, it is possible to capture the large scale components of the solution for a class of hyperbolic equations without resolving the small scale quantities on the computational grid. In the particle method studied in [6], the computational grid is formed by the intersection of different families of characteristic lines. The solution is updated along its own characteristic line. Since the other components of the solution are available at the same grid point, there is no need to use interpolation from one family of characteristics to another. This is important because interpolation may introduce large dissipative or dispersive errors which can damage the high frequency components of the solution. Because the particle method treats advection accurately and does not introduce dissipative or dispersive errors, it is an excellent method for computing multiscale solutions of partial differential equations. The sampling technique is used to avoid certain resonance between the coarse grid and the period of the small scale solution. By avoiding the resonant sampling of a coarse grid, one can approximate the correct mean and high order moments of the solution using only a coarse grid. On the other hand, forming the computational grid by the intersection of characteristic lines is a severe limitation of the method. When more than two equations are considered, the characteristics may never meet at one point. Special consideration is required in choosing the computational grid.

In this paper, we propose a modified particle method that overcomes the difficulty in choosing a computational grid. Our modified particle method retains most of the advantages in the original particle method and can be applied to hyperbolic systems with any number of families of characteristics. The modified method consists

\footnotetext{
*Received November 7, 2004; accepted for publication February 2, 2004.

${ }^{\dagger}$ Department of Mathematics, Stanford University, Stanford, CA 94305-2125, USA (van@math. stanford.edu).

¥Applied Mathematics, California Institute of Technology, Pasadena, CA 91125, USA (hou@acm. caltech.edu).
} 
of the following steps: 1) compute the characteristics corresponding to each component $u_{i}$ of an $n$-dimensional semilinear hyperbolic system, 2) update each scalar component $u_{i}(i=1, \ldots, n)$ along its own characteristics, 3$)$ interpolate to obtain all other components $u_{j}(j \neq i)$ of the solution to the $u_{i}$-characteristics, then return to Step 1 and continue. In order to avoid excessive numerical dissipation or dispersion, the interpolation scheme must be chosen carefully and the choice of an interpolation scheme depends on the semi-hyperbolic system being considered. In general, a high order interpolation scheme, such as a cubic spline interpolation or a spectral interpolation method, gives a better result than a lower order interpolation method. But for problems which do not have resonant interaction among high frequency components, such as the Carleman model, we found that lower order interpolation methods work equally well. This can be explained by our error analysis.

We apply our modified particle method to the variable coefficient Carleman model and the Broadwell model. We implement the modified particle method on a coarse grid without requiring that different families of characteristics intersect at the same point. Our numerical results indicate that the modified particle method preserves the main features (mean and variation of oscillations) of oscillatory solutions of Carleman and Broadwell models. In the case of the Carleman model with variable coefficients, we prove rigorously the convergence of the modified particle method essentially independent of the small scale. The proof relies on the homogenization theory developed for this model. As is the case for the original particle method, we need to take into account the cancellation of local truncation errors in time along the characteristics. Similar analysis applies to the non-resonant Broadwell model (see (6) with $\alpha$ being an irrational number). The convergence analysis for the original Broadwell model (4) is more subtle due to the resonant interaction among the three components of the solution. We have not yet obtained a convergence proof for this case. Nonetheless, our numerical results seem to indicate that the modified particle method applied to the original Broadwell model converges essentially independent of the small scale if a cubic spline or spectral interpolation is used. We plan to generalize the modified particle method to more complicated and more realistic hyperbolic systems in the future.

The paper is organized as follows. In Section 2 we introduce the Carleman and Broadwell models. Section 3 describes the modified particle method for the variable coefficient Carleman model. In Section 4 we study the convergence of the modified particle method proposed in Section 3. In Section 5 we present the numerical results of the modified particle method applied to both Carleman and Broadwell models. Some technical results are included in the Appendix.

\section{Carleman and Broadwell models.}

The Carleman model. The Carleman model is the simplest model of the discrete Boltzmann equations. It describes a one-dimensional gas whose molecules can only have two distinct velocities that change under collision [3]. The variables $u(x, t)$, $v(x, t)$ represent the number densities of molecules at point $x$ and time $t$ with velocities +1 and -1 , respectively. Based on mass conservation, the Carleman model is described by the following semi-linear hyperbolic system:

$$
\begin{aligned}
& \frac{\partial u}{\partial t}+\frac{\partial u}{\partial x}+u^{2}-v^{2}=0 \\
& \frac{\partial v}{\partial t}-\frac{\partial v}{\partial x}-u^{2}+v^{2}=0
\end{aligned}
$$


The model can be extended to include variable coefficients by replacing the constant velocities +1 and -1 with smooth functions $a(x, t)$ and $-b(x, t)$, respectively, satisfying

$$
0<a_{\min } \leq a(x, t) \leq a_{\max }<\infty, \quad 0<b_{\min } \leq b(x, t) \leq b_{\max }<\infty .
$$

The variable velocity Carleman model reads:

$$
\begin{aligned}
& \frac{\partial u}{\partial t}+a(x, t) \frac{\partial u}{\partial x}+u^{2}-v^{2}=0 \\
& \frac{\partial v}{\partial t}-b(x, t) \frac{\partial v}{\partial x}-u^{2}+v^{2}=0 .
\end{aligned}
$$

We consider initial data of multiple scale type:

$$
u(x, 0)=u_{0}\left(x, \frac{x}{\epsilon}\right), \quad v(x, 0)=v_{0}\left(x, \frac{x}{\epsilon}\right),
$$

where $u_{0}(x, y)$ and $v_{0}(x, y)$ are assumed to be 1 -periodic in $y$.

The high frequency components in the solution of Carleman equations (1),(3) or (2), (3), interact through the nonlinear terms and create low frequency components that affect the averaged solutions. The homogenization of the Carleman system has been studied in [12] and [6].

The Broadwell model. The $1 D$ Broadwell model is given by the following semi-linear hyperbolic system:

$$
\begin{aligned}
& \frac{\partial u}{\partial t}+\frac{\partial u}{\partial x}+u v-w^{2}=0 \\
& \frac{\partial v}{\partial t}-\frac{\partial v}{\partial x}+u v-w^{2}=0 \\
& \frac{\partial w}{\partial t}-u v+w^{2}=0 .
\end{aligned}
$$

The set of equations (4) represents a reduced version of the full Broadwell system (see [2]) that describes a three-dimensional model of a rarefied gas in which particles travel in either direction along a coordinate axis. In $(4), u(x, t), v(x, t)$ and $w(x, t)$ represent the number density of particles with velocity $(1,0,0),(-1,0,0)$ and $(0,1,0)$, respectively. To obtain (4) from the full Broadwell system one needs to assume that the number densities of particles moving in either direction are independent of $y, z$. Moreover, we assume that the number densities of particles moving in the negative $y$ direction or in either $z$ direction are equal to $w$.

We consider oscillatory initial data:

$$
u(x, 0)=u_{0}\left(x, \frac{x}{\epsilon}\right), \quad v(x, 0)=v_{0}\left(x, \frac{x}{\epsilon}\right), \quad w(x, 0)=w_{0}\left(x, \frac{x}{\epsilon}\right),
$$

where $u_{0}(x, y), v_{0}(x, y)$ and $w_{0}(x, y)$ are 1 -periodic in $y$.

The nonlinear interaction between the various components of the system (4) is very intricate $[8,11]$. The high frequency components of $u$ and $v$ interact and create order $O(1)$ oscillations on $w$ even in the limit $\epsilon \rightarrow 0$. The $w^{2}$ term will then generate low frequency contribution to $u$ and $v$, thus affecting the average of the solution (see the homogenized equations (7)).

In [8], the authors introduce an additional convection term in the last equation (4c) of the Broadwell model in order to study how oscillatory solutions depend on 
velocity coefficients. The modified system is referred to as the generalized Broadwell model and it reads

$$
\begin{array}{r}
\frac{\partial u}{\partial t}+\frac{\partial u}{\partial x}+u v-w^{2}=0 \\
\frac{\partial v}{\partial t}-\frac{\partial v}{\partial x}+u v-w^{2}=0 \\
\frac{\partial w}{\partial t}+\alpha \frac{\partial w}{\partial x}-u v+w^{2}=0
\end{array}
$$

The behavior of the solutions $u_{\epsilon}, v_{\epsilon}$ and $w_{\epsilon}$ of (6) as $\epsilon \rightarrow 0$ is very sensitive to the coefficient $\alpha$. Two different sets of effective equations are obtained depending on whether $\alpha$ is a rational or an irrational number. They are given below.

Case $I: \alpha=m / n$ ( $m$ and $n$ mutually prime); the homogenized equations for (6) are

$$
\begin{aligned}
& \frac{\partial U}{\partial t}+\frac{\partial U}{\partial x}+U \int_{0}^{1} V d y-\int_{0}^{1} W^{2} d y=0 \\
& \frac{\partial V}{\partial t}-\frac{\partial V}{\partial x}+V \int_{0}^{1} U d y-\int_{0}^{1} W^{2} d y=0 \\
& \frac{\partial W}{\partial t}+\alpha \frac{\partial W}{\partial x}+W^{2} \\
& \quad-\frac{1}{n} \int_{0}^{n} U\left(x, y+\left(\frac{m}{n}-1\right) z, t\right) V\left(x, y+\left(\frac{m}{n}+1\right) z, t\right) d z=0 .
\end{aligned}
$$

If $m=0$, then $n=1$ in $(7 \mathrm{c})$.

Case II: $\alpha$ is an irrational number; the homogenized equations for (6) are

$$
\begin{aligned}
& \frac{\partial U}{\partial t}+\frac{\partial U}{\partial x}+U \int_{0}^{1} V d y-\int_{0}^{1} W^{2} d y=0 \\
& \frac{\partial V}{\partial t}-\frac{\partial V}{\partial x}+V \int_{0}^{1} U d y-\int_{0}^{1} W^{2} d y=0 \\
& \frac{\partial W}{\partial t}+\alpha \frac{\partial W}{\partial x}+W^{2}-\left(\int_{0}^{1} U(x, y, t) d y\right)\left(\int_{0}^{1} V(x, y, t) d y\right)=0 .
\end{aligned}
$$

The following homogenization theorem is stated and proved in [8]:

THEOREM 2.1. For smooth and bounded non-negative initial data, the solutions of (6) and (5) converge to those of the corresponding homogenized equations strongly in $L^{\infty}$-norm,

$$
\begin{aligned}
u_{\epsilon}(x, t)-U\left(x, \frac{x-t}{\epsilon}, t\right) & \rightarrow 0 \quad \text { as } \epsilon \rightarrow 0, \\
v_{\epsilon}(x, t)-V\left(x, \frac{x+t}{\epsilon}, t\right) & \rightarrow 0 \quad \text { as } \epsilon \rightarrow 0, \\
w_{\epsilon}(x, t)-W\left(x, \frac{x-\alpha t}{\epsilon}, t\right) & \rightarrow 0 \quad \text { as } \epsilon \rightarrow 0 \quad \text { for } 0 \leq t \leq T .
\end{aligned}
$$

Moreover,

$$
\left|u_{\epsilon}(x, t)-U\left(x, \frac{x-t}{\epsilon}, t\right)\right| \leq C \epsilon, \quad \text { for all } x, t
$$




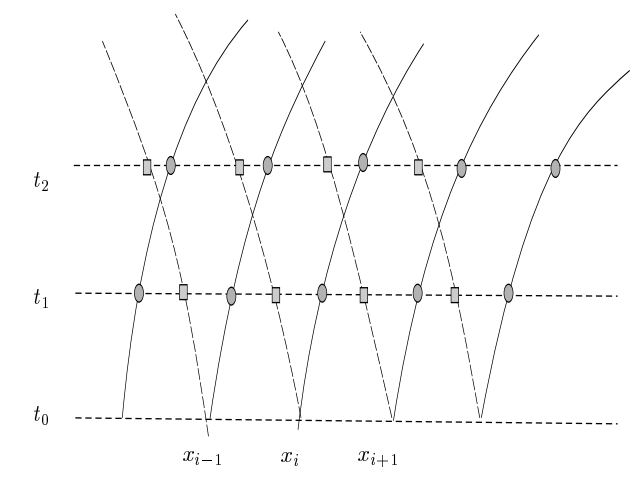

FIG. 1. The grid used for the modified Lagrangian method

where the constant $C$ is independent of $\epsilon$. Similar inequalities hold for $v_{\epsilon}, V$ and $w_{\epsilon}, W$.

3. A modified particle method. In this section we consider a modified particle method for semi-linear hyperbolic systems. The method in principle works for any number of families of characteristics and in any dimension.

The modified particle method. A diagram representing the computational grid for a $2 \times 2$ system is presented in Figure 1 . We will make use of this schematic diagram to describe the method for the variable coefficient Carleman model (2) with initial data (3).

For the Carleman system (2), the particle paths moving to the right in Figure 1 correspond to the $u$-characteristics and the ones moving to the left correspond to the $v$-characteristics.

The numerical scheme is initialized at $t=0$ by

$$
u_{i}^{0}=u_{0}\left(x_{i}, \frac{x_{i}}{\epsilon}\right), \quad v_{i}^{0}=v_{0}\left(x_{i}, \frac{x_{i}}{\epsilon}\right) .
$$

Here, $x_{i}=i \Delta x$ with $i \in \mathbb{Z}$ are equally spaced gridpoints, $\Delta x$ is the mesh size. They represent the Lagrangian coordinates (labels on the particles). To update the solution we use a fixed timestep $\Delta t$ and a sequence of discrete times $t_{n}=n \Delta t$.

Approximate characteristics. At each timestep in our modified particle method, we solve numerically the characteristic equations corresponding to each component of (2):

$$
\frac{d \Phi(s ; t, x)}{d s}=a(\Phi(s ; t, x), s), \quad \Phi(t ; t, x)=x
$$

and

$$
\frac{d \Psi(s ; t, x)}{d s}=-b(\Psi(s ; t, x), s), \quad \Psi(t ; t, x)=x .
$$

Here, $\Phi(s ; t, x)$ represents the location of a particle at time $s$ occupying the position $x$ at time $t$. 
Let $\tilde{x}_{i, u}^{n}$ and $\tilde{x}_{i, v}^{n}$ denote the approximations of $\Phi\left(t_{n} ; 0, x_{i}\right)$ and $\Psi\left(t_{n} ; 0, x_{i}\right)$, respectively. In the numerical scheme we use an explicit forward Euler method to compute the numerical characteristics:

$$
\begin{aligned}
& \tilde{x}_{i, u}^{n+1}=\tilde{x}_{i, u}^{n}+\Delta t a\left(\tilde{x}_{i, u}^{n}, t_{n}\right), \\
& \tilde{x}_{i, v}^{n+1}=\tilde{x}_{i, v}^{n}-\Delta t b\left(\tilde{x}_{i, v}^{n}, t_{n}\right) .
\end{aligned}
$$

In Figure 1 we represent schematically the points $\tilde{x}_{i, u}^{n}$ and $\tilde{x}_{i, v}^{n}($ for $n=1,2)$ with filled ellipses and filled rectangles, respectively. We use a particle method to update the initial values $u_{i}^{0}, v_{i}^{0}$ along the numerical $u, v$ characteristics, respectively. To update from $t=t_{0}$ to $t=t_{1}$, we compute

$$
\begin{aligned}
& \tilde{u}_{i}^{1}=u_{i}^{0}+\Delta t\left(\left(v_{i}^{0}\right)^{2}-\left(u_{i}^{0}\right)^{2}\right), \\
& \tilde{v}_{i}^{1}=v_{i}^{0}+\Delta t\left(\left(u_{i}^{0}\right)^{2}-\left(v_{i}^{0}\right)^{2}\right) .
\end{aligned}
$$

The values $\tilde{u}_{i}^{1}$ are evaluated at $\tilde{x}_{i, u}^{1}$ (the filled ellipses) and the values $\tilde{v}_{i}^{1}$ are evaluated at $\tilde{x}_{i, v}^{1}$ (the filled rectangles).

Interpolation. Consider an interpolation operator $\widetilde{\mathcal{I}}_{n}: l^{\infty} \rightarrow l^{\infty}$. The operator $\widetilde{\mathcal{I}}_{n}$ takes the components of a sequence in $l^{\infty}$ (considered to be a discrete function evaluated at the points $\tilde{x}_{i, v}^{n}, i \in \mathbb{Z}$ ) and returns their interpolated values at $\tilde{x}_{i, u}^{n}, i \in \mathbb{Z}$.

We can also consider the interpolation operator that takes an $l^{\infty}$ sequence regarded as a discrete function evaluated at $\tilde{x}_{i, u}^{n}, i \in \mathbb{Z}$, and returns the interpolated values at $\tilde{x}_{i, v}^{n}, i \in \mathbb{Z}$. We use the same symbol $\widetilde{\mathcal{I}}_{n}$ for the two interpolation operators, hoping that it is clear from the context which one we refer to.

The modified particle algorithm is given by (12) and

$$
\begin{aligned}
& \tilde{u}_{i}^{n+1}=\tilde{u}_{i}^{n}+\Delta t\left(\widetilde{\mathcal{I}}_{n}\left(\tilde{v}^{n}\right)^{2}{ }_{i}-\left(\tilde{u}_{i}^{n}\right)^{2}\right), \\
& \tilde{v}_{i}^{n+1}=\tilde{v}_{i}^{n}+\Delta t\left(\widetilde{\mathcal{I}}_{n}\left(\tilde{u}^{n}\right)^{2}{ }_{i}-\left(\tilde{v}_{i}^{n}\right)^{2}\right),
\end{aligned}
$$

where $\tilde{u}_{i}^{n}$, $\tilde{v}_{i}^{n}$ denote the numerical solution evaluated at $\tilde{x}_{i, u}^{n}, \tilde{x}_{i, v}^{n}$, respectively. We denote by $\tilde{u}^{n}, \tilde{v}^{n}$ the $l^{\infty}$ sequences with components $\tilde{u}_{i}^{n}, \tilde{v}_{i}^{n}$, respectively, and by $\widetilde{\mathcal{I}}_{n}\left(\tilde{u}^{n}\right)^{2}{ }_{i}$ and $\widetilde{\mathcal{I}}_{n}\left(\tilde{v}^{n}\right)^{2}{ }_{i}, i \in \mathbb{Z}$, the $i$-th components of the sequences $\widetilde{\mathcal{I}}_{n}\left(\tilde{u}^{n}\right)^{2}$ and $\widetilde{\mathcal{I}}_{n}\left(\tilde{v}^{n}\right)^{2}$, respectively.

In summary, the modified particle method consists of the following steps: 1) compute the numerical characteristics, 2) update the $i$ th $(i=1, \ldots, n)$ component of the solution along its own numerical characteristics, 3 ) interpolate other components of the solution from their own characteristic points to the $i$ th characteristic points and return to Step 1 for the next timestep.

Interpolation operator. In our numerical tests we use several interpolation procedures: linear, cubic spline, and convolution with a discrete delta function. It is clear that all these interpolation operators are linear. In the convergence proof to be presented in Section 4, we consider the case when the interpolation is done by a convolution with a discrete delta function.

Let $\theta_{\sigma}(x)$ be a smooth mollifier of the delta function $\delta(x)$ defined as follows:

$$
\theta_{\sigma}(x)=\frac{1}{\sigma} \theta\left(\frac{x}{\sigma}\right) \quad \text { with } \sigma=\Delta x^{\beta}, \quad 0<\beta<1,
$$


where $\Delta x$ is the grid size of the initial Lagrangian particle positions, and $\theta$ is a $C^{1}$ shape function with compact support normalized such that $\int \theta(x) d x=1$. An example of such shape function is the "cosine function"

$$
\theta(x)= \begin{cases}\frac{1}{2}(1+\cos (\pi x)) & |x|<1 \\ 0 & |x| \geq 1\end{cases}
$$

We consider for instance the interpolated sequence $\widetilde{\mathcal{I}}_{n}\left(\tilde{v}^{n}\right)^{2}$. Using the discrete convolution interpolation, we approximate the $i$-th component of the sequence $\widetilde{\mathcal{I}}_{n}\left(\tilde{v}^{n}\right)^{2}$ by

$$
\widetilde{\mathcal{I}}_{n}\left(\tilde{v}^{n}\right)_{i}^{2}=\Delta x \sum_{j} \theta_{\sigma}\left(\tilde{x}_{i, u}^{n}-\tilde{x}_{j, v}^{n}\right)\left(\tilde{v}_{j}^{n}\right)^{2} \frac{\partial \Psi}{\partial x}\left(t_{n} ; 0, x_{j}\right) .
$$

The values $\frac{\partial \Psi}{\partial x}\left(t_{n} ; 0, x_{j}\right)$ are computed numerically, by discretizing:

$$
\frac{d}{d t}\left(\frac{\partial \Psi}{\partial x}\right)=-\frac{\partial b}{\partial x} \frac{\partial \Psi}{\partial x},\left.\quad \frac{\partial \Psi}{\partial x}\right|_{t=0}=1
$$

The value in (17) is an approximation of

$$
\begin{aligned}
& \int_{\mathbb{R}} \theta_{\sigma}\left(\Phi\left(t_{n} ; 0, x_{i}\right)-\Psi\left(t_{n} ; 0, y\right)\right) v^{2}\left(\Psi\left(t_{n} ; 0, y\right), t_{n}\right) \frac{\partial \Psi}{\partial y}\left(t_{n} ; 0, y\right) d y \\
& =\int_{\mathbb{R}} \theta_{\sigma}\left(\left(\Phi\left(t_{n} ; 0, x_{i}\right)-z\right) v^{2}\left(z, t_{n}\right) d z\right.
\end{aligned}
$$

which, in turn, approximates

$$
\int_{\mathbb{R}} \delta\left(\left(\Phi\left(t_{n} ; 0, x_{i}\right)-z\right) v^{2}\left(z, t_{n}\right) d z=v^{2}\left(\left(\Phi\left(t_{n} ; 0, x_{i}\right), t_{n}\right) .\right.\right.
$$

The approximation of $\widetilde{\mathcal{I}}_{n}\left(\tilde{u}^{n}\right)^{2}$ is defined similarly.

In $[1$, Section 4], the authors present several discrete approximations of the delta function and discuss the order of the approximation error in using the corresponding discrete convolutions as interpolation. Their study, however, considers only interpolation of discrete values $f\left(x_{j}\right)$ evaluated at the equally spaced grid points $x_{j}=j \Delta x$, $j \in \mathbb{Z}$.

In the Appendix, we present a convergence analysis of the approximation error from the interpolation using a discrete convolution (17).

4. Convergence of the modified particle method. In this section we will prove the convergence of the modified particle scheme (12), (14) in the case when the interpolation is done by using discrete convolutions, as discussed in Section 3.

The following concept of convergence was introduced by Engquist in [5]:

DeFinition. Let $u^{n}$ represent the numerical approximation to $u$ at time $t_{n}$ $\left(t_{n}=n \Delta t, n=1,2, \ldots\right)$. The approximation $u^{n}$ converges to $u$ as $\Delta t \rightarrow 0$ essentially independent of $\epsilon$ if for any $\delta>0, T>0$ there exists a set $s\left(\epsilon, \Delta t_{0}\right) \subset\left(0, \Delta t_{0}\right)$ with measure $\left(s\left(\epsilon, \Delta t_{0}\right)\right) \geq(1-\delta) \Delta t_{0}$ such that

$$
\left\|u\left(\cdot, t_{n}\right)-u^{n}\right\| \leq \delta, \quad 0<t^{n} \leq T,
$$

for all $\Delta t \in s\left(\epsilon, \Delta t_{0}\right)$ and where $\Delta t_{0}$ is independent of $\epsilon$. 
REMARK. For the particle method investigated in [6], the set $s\left(\epsilon, \Delta t_{0}\right)$ excludes timesteps $\Delta t$ for which $\Delta t / \epsilon$ equals some rational numbers (see formula (21)).

The convergence proof presented in this section relies on the homogenization result available for the Cauchy problem (2)-(3) (see [6]). We will state it without proof:

THEOREM 4.1. The solution to (2) and (3) converges uniformly to that of the homogenized equations (19) and (20):

$$
\begin{aligned}
& u_{\epsilon}(x, t)-U\left(x, \frac{\Phi(0 ; t, x)}{\epsilon}, t\right) \rightarrow 0 \quad \text { as } \epsilon \rightarrow 0, \\
& v_{\epsilon}(x, t)-V\left(x, \frac{\Psi(0 ; t, x)}{\epsilon}, t\right) \rightarrow 0 \quad \text { as } \epsilon \rightarrow 0, \quad \text { for } 0 \leq t \leq T,
\end{aligned}
$$

where $\Phi(s ; t, x), \Psi(s ; t, x)$ are the characteristic maps defined by (10)-(11), and $U(x, y, t), V(x, y, t)$ are the solutions of the homogenized equations (19) and (20):

$$
\begin{gathered}
\frac{\partial U}{\partial t}+a(x, t) \frac{\partial U}{\partial x}+U^{2}-\int_{0}^{1} V^{2} d y=0, \\
\frac{\partial V}{\partial t}-b(x, t) \frac{\partial V}{\partial x}+V^{2}-\int_{0}^{1} U^{2} d y=0, \\
U(x, y, 0)=u_{0}(x, y), \quad V(x, y, 0)=v_{0}(x, y) .
\end{gathered}
$$

Let us state the following lemmas:

Lemma 4.1. The Cauchy problem (2)-(3) has bounded solutions in $C^{3}$. Moreover, if $0 \leq u_{0}, v_{0} \leq M<\infty$, then $0 \leq u(x, t), v(x, t) \leq M$ for $t>0$.

Lemma 4.2. Let $f(x), g(x, y)$ be $C^{1}$ functions. Assume further that $g(x, y)$ is 1periodic in $y$ and satisfies $\int_{0}^{1} g(x, y) d y=0$. Then, for any $\epsilon>0$ and for any constants $a$ and $b$, there exists a constant $C$ independent of $\epsilon$ such that

$$
\left|\int_{a}^{b} f(x) g\left(x, \frac{x}{\epsilon}\right) d x\right| \leq C \epsilon .
$$

LEMma 4.3. Let $f(x)$ and $g(x, y)$ be smooth functions with bounded derivatives. Assume further that $g(x, y)$ is 1 -periodic in $y$ and satisfies $\int_{0}^{1} g(x, y) d y=0$. Then, for $x_{k}=k \Delta x, 0 \leq m<n \leq T / \Delta x$, we have

$$
\left|\Delta x \sum_{k=m}^{n} f\left(x_{k}\right) g\left(x_{k}, \frac{x_{k}}{\epsilon}\right)\right| \rightarrow 0 \quad \text { as } \Delta x \rightarrow 0,
$$

essentially independent of $\epsilon$.

Lemmas 4.1, 4.2 and 4.3 are stated in [6]. Lemma 4.1 can be proved by following the proof of [9, Theorem 1]. Lemmas 4.2 and 4.3 are proved in [6]. 
REMARK. In [4], the authors explicitly state the set $s\left(\epsilon, \Delta x_{0}\right)$ corresponding to the essentially independent of $\epsilon$ convergence from Lemma 4.3. This set is given by

$$
s\left(\epsilon, \Delta x_{0}\right)=\left\{0<\Delta x \leq \Delta x_{0} ;(k \Delta x / \epsilon) \notin\left(i-\frac{\tau}{|k|^{3 / 2}}, i+\frac{\tau}{|k|^{3 / 2}}\right)\right\},
$$

with $i \in \mathbb{Z}$ and $0 \neq k \in \mathbb{Z}$. If $\Delta x \in s\left(\epsilon, \Delta x_{0}\right)$, the following estimate is proved in [4]:

$$
\left|\Delta x \sum_{k=m}^{n} f\left(x_{k}\right) g\left(x_{k}, \frac{x_{k}}{\epsilon}\right)\right| \leq C \frac{\Delta x}{\tau},
$$

where $C$ is independent of $\Delta x$ and $\epsilon$.

The next lemma is stated and proved by Engquist in [5]. It is an essential tool to prove convergence of the modified particle scheme (14).

Lemma 4.4. Let $E_{n}, R_{m, n} \in l^{\infty}$ with the properties:

$$
\begin{aligned}
E_{n+1} & =\left(P+\Delta t A_{n}\right) E_{n}+R_{n, n+1}, \quad n=0,1, \ldots, \\
E_{0} & =0,
\end{aligned}
$$

where the operators $P$ and $A_{n}$ satisfy

$$
\begin{gathered}
\|P\|=1, \quad\left\|A_{n}\right\| \leq C, \\
R_{n, n+1}+P R_{m, n}=R_{m, n+1}, \quad 0 \leq m<n .
\end{gathered}
$$

Then, $\left\|E_{n}\right\| \leq \exp \left(C t_{n}\right) \max _{0 \leq m<k \leq n}\left\|R_{m, k}\right\|$.

Step 1. We suppose first that the particle paths (the characteristic maps) $\Phi(s ; t, x), \Psi(s ; t, x)$ can be solved exactly from (10)-(11). We denote

$$
x_{i, u}^{n}=\Phi\left(t_{n} ; 0, x_{i}\right), \quad x_{i, v}^{n}=\Psi\left(t_{n} ; 0, x_{i}\right) .
$$

Hence, there is no error in generating the grid corresponding to the modified particle algorithm (14).

We denote by $u_{i}^{n}, v_{i}^{n}$ the solution of the modified particle algorithm when the characteristics are known analytically. The algorithm for solving $u_{i}^{n}, v_{i}^{n}$ is given by

$$
\begin{aligned}
& u_{i}^{n+1}=u_{i}^{n}+\Delta t\left(\mathcal{I}_{n}\left(v^{n}\right)^{2}{ }_{i}-\left(u_{i}^{n}\right)^{2}\right), \\
& v_{i}^{n+1}=v_{i}^{n}+\Delta t\left(\mathcal{I}_{n}\left(u^{n}\right)^{2}{ }_{i}-\left(v_{i}^{n}\right)^{2}\right) .
\end{aligned}
$$

In (24a), the interpolation operator $\mathcal{I}_{n}: l^{\infty} \rightarrow l^{\infty}$ takes the components of a sequence in $l^{\infty}\left(\right.$ regarded as a discrete function evaluated at the points $\left.x_{i, v}^{n}=\Psi\left(t_{n} ; 0, x_{i}\right), i \in \mathbb{Z}\right)$ and returns their interpolated values at $x_{i, u}^{n}=\Phi\left(t_{n} ; 0, x_{i}\right), i \in \mathbb{Z}$. A similar statement, with the roles of $u$ and $v$ interchanged, can be made about the interpolation operator used in (24b). For convenience, we use the same notation $\mathcal{I}_{n}$ for the two operators.

LEMma 4.5. Let $u_{i}^{n}, v_{i}^{n}$ be the solution of the modified particle algorithm (24) with initial values given by

$$
u_{i}^{0}=u_{0}\left(x_{i}, \frac{x_{i}}{\epsilon}\right), \quad v_{i}^{0}=v_{0}\left(x_{i}, \frac{x_{i}}{\epsilon}\right) .
$$

If the initial data are bounded and the mollifier function $\theta_{\sigma}$ in the discrete convolution is non-negative, then there exists a constant $M$ such that for $\Delta t \leq 1 /(2 M)$, we have $0 \leq u_{i}^{n}, v_{i}^{n} \leq M$ for $n=1,2, \ldots$. 
The proof of Lemma 4.5 will be deferred to the Appendix.

TheOrem 4.2. Assume that the grid points $x_{i, u}^{n}$ and $x_{i, v}^{n}$ have been solved exactly. Then, the solution $u_{i}^{n}, v_{i}^{n}$ of the modified particle algorithm (24) with initial values given by (25) converges in the maximum norm to the solution $u, v$ of the Cauchy problem (2)-(3) as $\Delta x, \Delta t \rightarrow 0$, essentially independent of $\epsilon$.

Proof. We will prove the theorem in the case when the interpolation is done by means of discrete convolutions.

Integrating (2) along the characteristics gives

$$
\begin{aligned}
& u\left(x_{i, u}^{n+1}, t_{n+1}\right)=u\left(x_{i, u}^{n}, t_{n}\right)+\int_{t_{n}}^{t_{n+1}}\left[v^{2}\left(\Phi\left(s ; 0, x_{i}\right), s\right)-u^{2}\left(\Phi\left(s ; 0, x_{i}\right), s\right)\right] d s, \\
& v\left(x_{i, v}^{n+1}, t_{n+1}\right)=v\left(x_{i, v}^{n}, t_{n}\right)+\int_{t_{n}}^{t_{n+1}}\left[u^{2}\left(\Psi\left(s ; 0, x_{i}\right), s\right)-v^{2}\left(\Psi\left(s ; 0, x_{i}\right), s\right)\right] d s .
\end{aligned}
$$

Further we denote by $e^{n}$ and $f^{n}$ the errors in the approximation of $u$ and $v$ along their respective characteristics:

$$
e_{i}^{n}=u\left(x_{i, u}^{n}, t_{n}\right)-u_{i}^{n}, \quad f_{i}^{n}=v\left(x_{i, v}^{n}, t_{n}\right)-v_{i}^{n} .
$$

We denote by $v\left(x_{\cdot, v}^{n}, t_{n}\right), u\left(x_{\cdot, u}^{n}, t_{n}\right) \in l^{\infty}$ the sequences whose $i$-th components are $v\left(x_{i, v}^{n}, t_{n}\right), u\left(x_{i, u}^{n}, t_{n}\right), i \in \mathbb{Z}$, respectively.

Subtracting (24a) from (26a) we obtain

$$
\begin{aligned}
e_{i}^{n+1}= & e_{i}^{n}+\Delta t\left[\mathcal{I}_{n} v^{2}\left(x_{\cdot, v}^{n}, t_{n}\right)_{i}-\mathcal{I}_{n}\left(v^{n}\right)^{2}{ }_{i}\right]-\Delta t\left[u^{2}\left(x_{i, u}^{n}, t_{n}\right)-\left(u_{i}^{n}\right)^{2}\right] \\
& +\int_{t_{n}}^{t_{n+1}} v^{2}\left(\Phi\left(s ; 0, x_{i}\right), s\right) d s-\Delta t \mathcal{I}_{n} v^{2}\left(x_{\cdot, v}^{n}, t_{n}\right)_{i} \\
& -\left[\int_{t_{n}}^{t_{n+1}} u^{2}\left(\Phi\left(s ; 0, x_{i}\right), s\right) d s-\Delta t u^{2}\left(x_{i, u}^{n}, t_{n}\right)\right] .
\end{aligned}
$$

Similarly, subtracting (24b) from (26b) we get

$$
\begin{aligned}
f_{i}^{n+1}= & f_{i}^{n}+\Delta t\left[\mathcal{I}_{n} u^{2}\left(x_{\cdot, u}^{n}, t_{n}\right)_{i}-\mathcal{I}_{n}\left(u^{n}\right)^{2}{ }_{i}\right]-\Delta t\left[v^{2}\left(x_{i, v}^{n}, t_{n}\right)-\left(v_{i}^{n}\right)^{2}\right] \\
& +\int_{t_{n}}^{t_{n+1}} u^{2}\left(\Psi\left(s ; 0, x_{i}\right), s\right) d s-\Delta t \mathcal{I}_{n} u^{2}\left(x_{\cdot, u}^{n}, t_{n}\right)_{i} \\
& -\left[\int_{t_{n}}^{t_{n+1}} v^{2}\left(\Psi\left(s ; 0, x_{i}\right), s\right) d s-\Delta t v^{2}\left(x_{i, v}^{n}, t_{n}\right)\right] .
\end{aligned}
$$

By using the linearity of the interpolation operator and the boundedness properties given by Lemmas 4.1 and 4.5, equations (27) and (28) can be written as:

$$
\begin{aligned}
& e_{i}^{n+1}=e_{i}^{n}-\Delta t \alpha_{i}^{n} e_{i}^{n}+\Delta t \mathcal{I}_{n}\left(\beta^{n} f^{n}\right)_{i}+J_{+, i}^{n, n+1}\left(v^{2}\right)-I_{+, i}^{n, n+1}\left(u^{2}\right), \\
& f_{i}^{n+1}=f_{i}^{n}-\Delta t \tilde{\alpha}_{i}^{n} f_{i}^{n}+\Delta t \mathcal{I}_{n}\left(\tilde{\beta}^{n} e^{n}\right)_{i}+J_{-, i}^{n, n+1}\left(u^{2}\right)-I_{-, i}^{n, n+1}\left(v^{2}\right),
\end{aligned}
$$


respectively. Here,

$$
\begin{aligned}
& J_{+, i}^{m, n}(w)=\int_{t_{m}}^{t_{n}} w\left(\Phi\left(s ; 0, x_{i}\right), s\right) d s-\Delta t \sum_{k=m}^{n-1} \mathcal{I}_{k} w\left(x_{\cdot, v}^{k}, t_{k}\right)_{i}, \\
& I_{+, i}^{m, n}(w)=\int_{t_{m}}^{t_{n}} w\left(\Phi\left(s ; 0, x_{i}\right), s\right) d s-\Delta t \sum_{k=m}^{n-1} w\left(x_{i, u}^{k}, t_{k}\right), \\
& J_{-, i}^{m, n}(w)=\int_{t_{m}}^{t_{n}} w\left(\Psi\left(s ; 0, x_{i}\right), s\right) d s-\Delta t \sum_{k=m}^{n-1} \mathcal{I}_{k} w\left(x_{\cdot, u}^{k}, t_{k}\right)_{i}, \\
& I_{-, i}^{m, n}(w)=\int_{t_{m}}^{t_{n}} w\left(\Psi\left(s ; 0, x_{i}\right), s\right) d s-\Delta t \sum_{k=m}^{n-1} w\left(x_{i, v}^{k}, t_{k}\right),
\end{aligned}
$$

and $0 \leq \alpha_{i}^{n}, \beta_{i}^{n}, \tilde{\alpha}_{i}^{n}, \tilde{\beta}_{i}^{n} \leq 2 M, i \in \mathbb{Z}$.

Define:

$$
\begin{aligned}
E_{n} & =\left(\ldots, e_{i}^{n}, f_{i}^{n}, \ldots\right), \\
A_{n} E_{n} & =\left(\ldots,-\alpha_{i}^{n} e_{i}^{n}+\mathcal{I}_{n}\left(\beta^{n} f^{n}\right)_{i},-\tilde{\alpha}_{i}^{n} f_{i}^{n}+\mathcal{I}_{n}\left(\tilde{\beta}^{n} e^{n}\right)_{i}, \ldots\right), \\
R_{m, n} & =\left(\ldots, J_{+, i}^{m, n}\left(v^{2}\right)-I_{+, i}^{m, n}\left(u^{2}\right), J_{-, i}^{m, n}\left(u^{2}\right)-I_{-, i}^{m, n}\left(v^{2}\right), \ldots\right) .
\end{aligned}
$$

Note that the operator $A_{n}$ is bounded as a map from $l^{\infty}$ to $l^{\infty}$. This is a consequence of the boundedness of the interpolation operator and the boundedness of the sequences $\alpha^{n}, \beta^{n}, \tilde{\alpha}^{n}$ and $\tilde{\beta}^{n}$. Moreover, $R_{m, n}$ satisfy

$$
R_{n, n+1}+R_{m, n}=R_{m, n+1}, \quad 0 \leq m<n .
$$

Let $P=\mathrm{Id}$ and define $E_{n}, A_{n}, R_{m, n}$ by (35)-(37). By applying Lemma 4.4 to $P, E_{n}$, $A_{n}, R_{m, n}$ defined above, we prove convergence of the modified particle method if we can show $\left\|R_{m, k}\right\| \rightarrow 0$ as $\Delta x, \Delta t \rightarrow 0$ essentially independent of $\epsilon$.

There are two generic terms in (37), i.e. $J_{+, i}^{m, n}\left(v^{2}\right)-I_{+, i}^{m, n}\left(u^{2}\right)$ and $J_{-, i}^{m, n}\left(u^{2}\right)-$ $I_{-, i}^{m, n}\left(v^{2}\right)$. We will analyze only the first term. The analysis for the second term is completely analogous.

Observe that

$$
\left|\frac{d}{d s} u^{2}\left(\Phi\left(s ; 0, x_{i}\right)\right)\right|=2\left|u v^{2}-u^{3}\right| \leq 4 M^{3} .
$$

A classical error estimate [10] gives:

$$
\left|I_{+, i}^{m, n}\left(u^{2}\right)\right| \leq C \Delta t \rightarrow 0 \quad \text { as } \Delta t \rightarrow 0 .
$$

For the term $J_{+, i}^{m, n}\left(v^{2}\right)$, we consider two cases. In both cases we take $\Delta t \sim O(\Delta x)$. Case 1. $\epsilon>\Delta x^{\beta / 2}$, where $0<\beta<1$ is defined in (15).

We write:

$$
\begin{aligned}
J_{+, i}^{m, n}\left(v^{2}\right)= & \int_{t_{m}}^{t_{n}} v^{2}\left(\Phi\left(s ; 0, x_{i}\right), s\right) d s-\Delta t \sum_{k=m}^{n-1} v^{2}\left(x_{i, u}^{k}, t_{k}\right) \\
& +\Delta t \sum_{k=m}^{n-1} v^{2}\left(x_{i, u}^{k}, t_{k}\right)-\Delta t \sum_{k=m}^{n-1} \mathcal{I}_{k} v^{2}\left(x_{\cdot, v}^{k}, t_{k}\right)_{i} .
\end{aligned}
$$


We have:

$$
\begin{aligned}
\left|\int_{t_{m}}^{t_{n}} v^{2}\left(\Phi\left(s ; 0, x_{i}\right), s\right) d s-\Delta t \sum_{k=m}^{n-1} v^{2}\left(x_{i, u}^{k}, t_{k}\right)\right| & \leq C \Delta t \max _{0 \leq s \leq T}\left|\frac{d}{d s} v^{2}\left(\Phi\left(s ; 0, x_{i}\right), s\right)\right| \\
& \leq \tilde{C} \frac{\Delta x}{\epsilon}<\tilde{C} \Delta x^{1-\beta / 2}
\end{aligned}
$$

On the other hand, the interpolation error estimate (71) from the Appendix gives:

$$
\left|v^{2}\left(x_{i, u}^{k}, t_{k}\right)-\mathcal{I}_{k} v^{2}\left(x_{\cdot, v}^{k}, t_{k}\right)_{i}\right| \leq C_{1} \frac{\Delta x}{\sigma}+C_{2} \frac{\sigma}{\epsilon},
$$

where $C_{1}$ and $C_{2}$ are constants. Recall that $\sigma=\Delta x^{\beta}$ from (15). We have

$$
\left|\Delta t \sum_{k=m}^{n-1}\left[v^{2}\left(x_{i, u}^{k}, t_{k}\right)-\mathcal{I}_{k} v^{2}\left(x_{\cdot, v}^{k}, t_{k}\right)_{i}\right]\right| \leq C_{1}\left(\Delta x^{1-\beta}\right)+C_{2}\left(\Delta x^{\beta / 2}\right) .
$$

Now, (38)-(40) prove the desired result in Case 1.

Case 2. $\epsilon \leq \Delta x^{\beta / 2}$.

By Theorem 4.1 we can replace $v(x, t)$ by $V(x, \Psi(0 ; t, x) / \epsilon, t)$. We split $V^{2}(x, y, t)$ into a mean and a fluctuation part as follows:

$$
V^{2}(x, y, t)=\left(\int_{0}^{1} V^{2}(x, y, t) d y\right)+\widetilde{V^{2}}(x, y, t) .
$$

It suffices to study $J_{+, i}^{m, n}\left(\widetilde{V^{2}}\right)$. Using the formula for the discrete convolution interpolation (see (17)), we have

$$
\begin{aligned}
& J_{+, i}^{m, n}\left(\widetilde{V^{2}}\right)=\int_{t_{m}}^{t_{n}} \widetilde{V^{2}}\left(\Phi\left(s ; 0, x_{i}\right), \frac{\Psi\left(0 ; s, \Phi\left(s ; 0, x_{i}\right)\right)}{\epsilon}, s\right) d s \\
& -\Delta t \sum_{k=m}^{n-1} \Delta x \sum_{j} \theta_{\sigma}\left(\Phi\left(t_{k} ; 0, x_{i}\right)-\Psi\left(t_{k} ; 0, x_{j}\right)\right) \widetilde{V^{2}}\left(\Psi\left(t_{k} ; 0, x_{j}\right), \frac{x_{j}}{\epsilon}, t_{k}\right) \frac{\partial \Psi}{\partial x}\left(t_{k} ; 0, x_{j}\right),
\end{aligned}
$$

where we have used $\Psi\left(0 ; t_{k}, \Psi\left(t_{k} ; 0, x_{j}\right)\right)=x_{j}$.

For a fixed $x_{i}$, define

$$
\begin{gathered}
h(s)=\Psi\left(0 ; s, \Phi\left(s ; 0, x_{i}\right)\right), \\
g(s, y)=\widetilde{V^{2}}\left(\Phi\left(s ; 0, x_{i}\right), y, s\right) .
\end{gathered}
$$

The first term in the right hand side (RHS) of (41) becomes:

$$
\int_{t_{m}}^{t_{n}} g\left(s, \frac{h(s)}{\epsilon}\right) d s .
$$

By changing the variable $h(s)=t$ (note that $h(s)$ is strictly increasing and hence, it has an inverse $h^{-1}(s)$ ), we have

$$
\int_{t_{m}}^{t_{n}} g\left(s, \frac{h(s)}{\epsilon}\right) d s=\int_{h\left(t_{m}\right)}^{h\left(t_{n}\right)} \frac{d h^{-1}(t)}{d t} g\left(h^{-1}(t), \frac{t}{\epsilon}\right) d t .
$$


Now we apply Lemma 4.2 to obtain:

$$
\left|\int_{t_{m}}^{t_{n}} \widetilde{V^{2}}\left(\Phi\left(s ; 0, x_{i}\right), \frac{\Psi\left(0 ; s, \Phi\left(s ; 0, x_{i}\right)\right)}{\epsilon}, s\right) d s\right| \leq C \epsilon \leq C \Delta x^{\beta / 2} .
$$

It remains to investigate the sum:

$$
\Delta t \sum_{k=m}^{n-1} \Delta x \sum_{j} \theta_{\sigma}\left(\Phi\left(t_{k} ; 0, x_{i}\right)-\Psi\left(t_{k} ; 0, x_{j}\right)\right) \widetilde{V^{2}}\left(\Psi\left(t_{k} ; 0, x_{j}\right), \frac{x_{j}}{\epsilon}, t_{k}\right) \frac{\partial \Psi}{\partial x}\left(t_{k} ; 0, x_{j}\right) .
$$

Due to the finite support of the function $\theta_{\sigma}$, the index $j$ in (45) satisfies

$$
\left|\Phi\left(t_{k} ; 0, x_{i}\right)-\Psi\left(t_{k} ; 0, x_{j}\right)\right| \leq \sigma .
$$

Interchange the order of the two summations. Then, the index $j$ will vary between $j=M$ and $j=N-1$ for some finite integers $M$ and $N$, and $t_{k}$ varies according to the inequality (46), for $j$ fixed. Denote by $I_{j}$ the set of indices $k$ that satisfy (46). Then, the sum (45) can be written as

$$
\Delta x \sum_{j=M}^{N-1} \Delta t \sum_{k \in I_{j}} \theta_{\sigma}\left(\Phi\left(t_{k} ; 0, x_{i}\right)-\Psi\left(t_{k} ; 0, x_{j}\right)\right) \widetilde{V^{2}}\left(\Psi\left(t_{k} ; 0, x_{j}\right), \frac{x_{j}}{\epsilon}, t_{k}\right) \frac{\partial \Psi}{\partial x}\left(t_{k} ; 0, x_{j}\right) .
$$

We denote the time continuous version of the term

$$
\Delta t \sum_{k \in I_{j}} \theta_{\sigma}\left(\Phi\left(t_{k} ; 0, x_{i}\right)-\Psi\left(t_{k} ; 0, x_{j}\right)\right) \widetilde{V^{2}}\left(\Psi\left(t_{k} ; 0, x_{j}\right), \frac{x_{j}}{\epsilon}, t_{k}\right) \frac{\partial \Psi}{\partial x}\left(t_{k} ; 0, x_{j}\right),
$$

by $w\left(x_{j}, \frac{x_{j}}{\epsilon}\right)$, where

$$
w\left(x, \frac{x}{\epsilon}\right)=\int_{I_{x}} \theta_{\sigma}\left(\Phi\left(t ; 0, x_{i}\right)-\Psi(t ; 0, x)\right) \widetilde{V^{2}}\left(\Psi(t ; 0, x), \frac{x}{\epsilon}, t\right) \frac{\partial \Psi}{\partial x}(t ; 0, x) d t,
$$

and

$$
I_{x}=\left\{t,\left|\Phi\left(t ; 0, x_{i}\right)-\Psi(t ; 0, x)\right| \leq \sigma\right\} .
$$

We write (47) as

$$
\begin{aligned}
\Delta x & \sum_{j=M}^{N-1} w\left(x_{j}, \frac{x_{j}}{\epsilon}\right)-\Delta x \sum_{j=M}^{N-1}\left[w\left(x_{j}, \frac{x_{j}}{\epsilon}\right)\right. \\
& \left.\quad-\Delta t \sum_{k \in I_{j}} \theta_{\sigma}\left(\Phi\left(t_{k} ; 0, x_{i}\right)-\Psi\left(t_{k} ; 0, x_{j}\right)\right) \widetilde{V^{2}}\left(\Psi\left(t_{k} ; 0, x_{j}\right), \frac{x_{j}}{\epsilon}, t_{k}\right) \frac{\partial \Psi}{\partial x}\left(t_{k} ; 0, x_{j}\right)\right] .
\end{aligned}
$$

We label $t_{k} \in I_{j}$ by $t_{1}, t_{2}, \ldots, t_{p_{j}}$. Clearly, $t_{k}, k=1, \ldots, p_{j}$ are also in the continuous set $I_{x_{j}}$. The measure of the set $I_{x_{j}} \backslash\left[t_{1}, t_{p_{j}}\right]$ is of order $O(\Delta t)$.

We also note that the length of the interval $\left[t_{1}, t_{p_{j}}\right]$ is of order $O(\sigma)$. To see this, we use a Taylor expansion

$$
\Phi\left(t_{p_{j}} ; 0, x_{i}\right)-\Psi\left(t_{p_{j}} ; 0, x_{j}\right)=\Phi\left(t_{1} ; 0, x_{i}\right)-\Psi\left(t_{1} ; 0, x_{j}\right)+\left(t_{p_{j}}-t_{1}\right)[a(\xi)+b(\eta)],
$$


where $\xi=\left(\Phi\left(\tilde{t} ; 0, x_{i}\right), \tilde{t}\right)$ and $\eta=\left(\Psi\left(t^{*} ; 0, x_{j}\right), t^{*}\right)$, with $\tilde{t}$ and $t^{*}$ being two intermediate points. Now we use

$$
\left|\Phi\left(t_{1} ; 0, x_{i}\right)-\Psi\left(t_{1} ; 0, x_{j}\right)\right| \leq \sigma
$$

and

$$
\left|\Phi\left(t_{p_{j}} ; 0, x_{i}\right)-\Psi\left(t_{p_{j}} ; 0, x_{j}\right)\right| \leq \sigma,
$$

to conclude

$$
0 \leq t_{p_{j}}-t_{1} \leq \frac{2 \sigma}{a(\xi)+b(\eta)} \leq \frac{2 \sigma}{a_{0}+b_{0}},
$$

where $a_{0}$ and $b_{0}$ are the minimum values of $a(x, t)$ and $b(x, t)$, respectively.

We now estimate (48). By Lemma 4.3, the first sum converges to zero essentially independent of $\epsilon$ since $w(x, y)$ has zero mean with respect to the second variable. As for the second sum, we study the term in the square bracket carefully. We have:

$$
\begin{aligned}
w & \left(x_{j}, \frac{x_{j}}{\epsilon}\right)-\Delta t \sum_{k \in I_{j}} \theta_{\sigma}\left(\Phi\left(t_{k} ; 0, x_{i}\right)-\Psi\left(t_{k} ; 0, x_{j}\right)\right) \widetilde{V^{2}}\left(\Psi\left(t_{k} ; 0, x_{j}\right), \frac{x_{j}}{\epsilon}, t_{k}\right) \frac{\partial \Psi}{\partial x}\left(t_{k} ; 0, x_{j}\right) \\
= & \int_{t_{1}}^{t_{p_{j}}} \theta_{\sigma}\left(\Phi\left(t ; 0, x_{i}\right)-\Psi\left(t ; 0, x_{j}\right)\right) \widetilde{V^{2}}\left(\Psi\left(t ; 0, x_{j}\right), \frac{x_{j}}{\epsilon}, t\right) \frac{\partial \Psi}{\partial x}\left(t ; 0, x_{j}\right) d t \\
& -\Delta t \sum_{k=1}^{p_{j}-1} \theta_{\sigma}\left(\Phi\left(t_{k} ; 0, x_{i}\right)-\Psi\left(t_{k} ; 0, x_{j}\right)\right) \widetilde{V^{2}}\left(\Psi\left(t_{k} ; 0, x_{j}\right), \frac{x_{j}}{\epsilon}, t_{k}\right) \frac{\partial \Psi}{\partial x}\left(t_{k} ; 0, x_{j}\right) \\
& +\int_{I_{x_{j}} \backslash\left[t_{1}, t_{p_{j}}\right]} \theta_{\sigma}\left(\Phi\left(t ; 0, x_{i}\right)-\Psi\left(t ; 0, x_{j}\right)\right) \widetilde{V^{2}}\left(\Psi\left(t ; 0, x_{j}\right), \frac{x_{j}}{\epsilon}, t\right) \frac{\partial \Psi}{\partial x}\left(t ; 0, x_{j}\right) d t \\
& -\Delta t \theta_{\sigma}\left(\Phi\left(t_{p_{j}} ; 0, x_{i}\right)-\Psi\left(t_{p_{j}} ; 0, x_{j}\right)\right) \widetilde{V^{2}}\left(\Psi\left(t_{p_{j}} ; 0, x_{j}\right), \frac{x_{j}}{\epsilon}, t_{p_{j}}\right) \frac{\partial \Psi}{\partial x}\left(t_{p_{j}} ; 0, x_{j}\right) .
\end{aligned}
$$

The first two terms in the RHS of (49) give the standard error in the rectangle rule approximation [10]. Denote the integrand by $f(t)$ :

$$
f(t)=\theta_{\sigma}\left(\Phi\left(t ; 0, x_{i}\right)-\Psi\left(t ; 0, x_{j}\right)\right) \widetilde{V^{2}}\left(\Psi\left(t ; 0, x_{j}\right), \frac{x_{j}}{\epsilon}, t\right) \frac{\partial \Psi}{\partial x}\left(t ; 0, x_{j}\right) .
$$

Recall that the function $\theta_{\sigma}$ is defined by (15). Since $\widetilde{V^{2}}\left(\Psi\left(t ; 0, x_{j}\right), \frac{x_{j}}{\epsilon}, t\right)$ and $\frac{\partial \Psi}{\partial x}\left(t ; 0, x_{j}\right)$ is a smooth function of $t$, we conclude that

$$
f^{\prime}(t)=O\left(\frac{1}{\sigma^{2}}\right),
$$

where the leading order term comes from the differentiation of $\theta_{\sigma}\left(\Phi\left(t ; 0, x_{i}\right)-\right.$ $\left.\Psi\left(t ; 0, x_{j}\right)\right)$ with respect to $t$. Thus the error estimate for the rectangle rule approximation implies that the difference of the first two terms in the RHS of (49) is of order:

$$
O\left(\frac{\Delta t\left(t_{p_{j}}-t_{1}\right)}{\sigma^{2}}\right)=O\left(\frac{\Delta t}{\sigma}\right) .
$$

Moreover, using the fact that

$$
\operatorname{measure}\left(I_{x_{j}} \backslash\left[t_{1}, t_{p_{j}}\right]\right)=O(\Delta t),
$$


and the boundedness of the integrand in the third term of the RHS of (49), we obtain

$$
\left|\int_{I_{x_{j}} \backslash\left[t_{1}, t_{p_{j}}\right]} \theta_{\sigma}\left(\Phi\left(t ; 0, x_{i}\right)-\Psi\left(t_{k} ; 0, x\right)\right) \widetilde{V^{2}}\left(\Psi(t ; 0, x), \frac{x}{\epsilon}, t\right) \frac{\partial \Psi}{\partial x}(t ; 0, x) d t\right| \leq C \frac{\Delta t}{\sigma} .
$$

Finally, it is easy to see that the fourth term in the RHS of (49) is also of order $O(\Delta t / \sigma)$.

We conclude that the expression in the left hand side (LHS) of (49) and hence, the expression in the square brackets in the second sum in (48), is of order $O\left(\frac{\Delta t}{\sigma}\right)$. From this result and the estimate (22) we infer that the expression in (48) is of order

$$
O\left(\frac{\Delta x}{\tau}\right)+O\left(\frac{\Delta t}{\sigma}\right) .
$$

We combine the above estimate for the term in (45) with estimate (44) to conclude that

$$
J_{+, i}^{m, n}\left(\widetilde{V^{2}}\right) \rightarrow 0 \quad \text { as } \Delta t \rightarrow 0,
$$

essentially independent of $\epsilon$. This completes the proof of Theorem 4.2.

Step 2. Now we consider the case when the particle paths are not known analytically. Let $\tilde{x}_{i, u}^{n}, \tilde{x}_{i, v}^{n}$ be the approximations of $x_{i, u}^{n}=\Phi\left(t_{n} ; 0, x_{i}\right)$ and $x_{i, v}^{n}=\Psi\left(t_{n} ; 0, x_{i}\right)$, $i \in \mathbb{Z}$, which are obtained by solving (12a)-(12b) respectively.

We continue to use $u_{i}^{n}, v_{i}^{n}$ to denote the solution of the modified particle algorithm (24) using the exact grid $x_{i, u}^{n}, x_{i, v}^{n}$, as in Step 1 . We denote by $\tilde{u}_{i}^{n}, \tilde{v}_{i}^{n}$ the solution of the modified particle algorithm (14) using the approximated grid $\tilde{x}_{i, u}^{n}, \tilde{x}_{i, v}^{n}$.

We first note that the proof of Lemma 4.5 can be extended to the solution of the modified particle algorithm (14). Thus, we can find a constant $\widetilde{M}>0$ such that for $\Delta t \leq 1 /(2 \widetilde{M})$, we have $0 \leq \tilde{u}_{i}^{n}, \tilde{v}_{i}^{n} \leq \widetilde{M}, i \in \mathbb{Z}$.

THEOREM 4.3. The solution $\tilde{u}_{i}^{n}, \tilde{v}_{i}^{n}$ of the modified particle algorithm (12), (14), with initial values given by (25), converges in the maximum norm to the solution $u$, $v$ of the Cauchy problem (2,3) as $\Delta x, \Delta t \rightarrow 0$, essentially independent of $\epsilon$.

Proof. The algorithm (12) is the forward Euler method, which is first order. We have:

$$
\begin{aligned}
x_{i, u}^{n}-\tilde{x}_{i, u}^{n}=O(\Delta t), & i \in \mathbb{Z}, \\
x_{i, v}^{n}-\tilde{x}_{i, v}^{n}=O(\Delta t), & i \in \mathbb{Z} .
\end{aligned}
$$

Denote

$$
e_{i}^{n}=\left|u_{i}^{n}-\tilde{u}_{i}^{n}\right|, \quad f_{i}^{n}=\left|v_{i}^{n}-\tilde{v}_{i}^{n}\right| .
$$

Subtracting (14a) from (24a) we obtain

$$
e_{i}^{n+1} \leq e_{i}^{n}+\Delta t\left|\mathcal{I}_{n}\left(v^{n}\right)^{2}{ }_{i}-\widetilde{\mathcal{I}}_{n}\left(\tilde{v}^{n}\right)^{2}{ }_{i}\right|+C_{1} \Delta t e_{i}^{n},
$$

with $C_{1}>0$. 
The interpolation using $\widetilde{\mathcal{I}}_{n}$ involves the numerical approximations of $\frac{\partial \Psi}{\partial x}\left(t_{n} ; 0, x_{j}\right)$ (see (17)) obtained by solving (18). Let $\left(\widetilde{\Psi}_{x}\right)_{j}^{n}$ denote the numerical solution of (18), using the forward Euler method. Then we have

$$
\frac{\partial \Psi}{\partial x}\left(t_{n} ; 0, x_{j}\right)-\left(\widetilde{\Psi}_{x}\right)_{j}^{n}=O(\Delta t) .
$$

We compute

$$
\begin{aligned}
& \mathcal{I}_{n}\left(v^{n}\right)^{2}{ }_{i}-\widetilde{\mathcal{I}}_{n}\left(\tilde{v}^{n}\right)^{2}{ }_{i} \\
&= \Delta x \sum_{j} \theta_{\sigma}\left(x_{i, u}^{n}-x_{j, v}^{n}\right)\left(v_{j}^{n}\right)^{2} \frac{\partial \Psi}{\partial x}\left(t_{n} ; 0, x_{j}\right)-\Delta x \sum_{j} \theta_{\sigma}\left(\tilde{x}_{i, u}^{n}-\tilde{x}_{j, v}^{n}\right)\left(\tilde{v}_{j}^{n}\right)^{2}\left(\widetilde{\Psi}_{x}\right)_{j}^{n} \\
&= \Delta x \sum_{j}\left[\theta_{\sigma}\left(x_{i, u}^{n}-x_{j, v}^{n}\right) \frac{\partial \Psi}{\partial x}\left(t_{n} ; 0, x_{j}\right)-\theta_{\sigma}\left(\tilde{x}_{i, u}^{n}-\tilde{x}_{j, v}^{n}\right)\left(\widetilde{\Psi}_{x}\right)_{j}^{n}\right]\left(v_{j}^{n}\right)^{2} \\
&+\Delta x \sum_{j} \theta_{\sigma}\left(\tilde{x}_{i, u}^{n}-\tilde{x}_{j, v}^{n}\right)\left(\widetilde{\Psi}_{x}\right)_{j}^{n}\left[\left(v_{j}^{n}\right)^{2}-\left(\tilde{v}_{j}^{n}\right)^{2}\right] .
\end{aligned}
$$

We estimate each term in the RHS of (51). Using the fact that the approximations of $x_{i, u}^{n}, x_{i, v}^{n}$ and $\frac{\partial \Psi}{\partial x}\left(t_{n} ; 0, x_{j}\right)$ are first order, the term in the square brackets in the first sum of the RHS of (51) can be estimated by

$$
\left|\theta_{\sigma}\left(x_{i, u}^{n}-x_{j, v}^{n}\right) \frac{\partial \Psi}{\partial x}\left(t_{n} ; 0, x_{j}\right)-\theta_{\sigma}\left(\tilde{x}_{i, u}^{n}-\tilde{x}_{j, v}^{n}\right)\left(\widetilde{\Psi}_{x}\right)_{j}^{n}\right| \leq C_{2}\left(\frac{\Delta t}{\sigma^{2}}\right) .
$$

Then we have

$$
\left|\Delta x \sum_{j}\left[\theta_{\sigma}\left(x_{i, u}^{n}-x_{j, v}^{n}\right) \frac{\partial \Psi}{\partial x}\left(t_{n} ; 0, x_{j}\right)-\theta_{\sigma}\left(\tilde{x}_{i, u}^{n}-\tilde{x}_{j, v}^{n}\right)\left(\widetilde{\Psi}_{x}\right)_{j}^{n}\right]\left(v_{j}^{n}\right)^{2}\right| \leq C_{3} \frac{\Delta t}{\sigma},
$$

where we have used the fact that the number of terms in the summation is of order $O(\sigma / \Delta x)$.

Regarding the second term in the RHS of (51), we have

$$
\left|\Delta x \sum_{j} \theta_{\sigma}\left(\tilde{x}_{i, u}^{n}-\tilde{x}_{j, v}^{n}\right)\left(\widetilde{\Psi}_{x}\right)_{j}^{n}\left[\left(v_{j}^{n}\right)^{2}-\left(\tilde{v}_{j}^{n}\right)^{2}\right]\right| \leq C_{4} \max _{j} f_{j}^{n},
$$

where we have used the boundedness properties of the solutions $v_{i}^{n}, \tilde{v}_{i}^{n}$.

Using the last two estimates for the RHS of (51), we obtain

$$
e_{i}^{n+1} \leq e_{i}^{n}+C_{1} \Delta t e_{i}^{n}+C_{4} \Delta t \max _{j} f_{j}^{n}+C_{3} \frac{\Delta t^{2}}{\sigma} .
$$

A similar inequality can be derived for $f_{i}^{n+1}$. Denote

$$
E_{n}=\max \left\{\max _{i} e_{i}^{n}, \max _{i} f_{i}^{n}\right\} .
$$

Then we have

$$
E_{n+1} \leq(1+C \Delta t) E_{n}+C \Delta t^{2} / \sigma,
$$

for a positive constant $C$. 
From (52) one can prove by induction the following inequality:

$$
E_{n} \leq C \Delta t\left(\sum_{j=0}^{n}(1+C \Delta t)^{j}\right) \Delta t / \sigma .
$$

Note that

$$
C \Delta t\left(\sum_{j=0}^{n}(1+C \Delta t)^{j}\right)=(1+C \Delta t)^{n}-1 \leq \exp (C T),
$$

which implies

$$
E_{n} \leq C_{5}\left(\frac{\Delta t}{\sigma}\right) .
$$

Now, the proof follows from the estimate (54) and Theorem 4.2.

REMARK. We remark that the same analysis applies to the generalized Broadwell model (6) with $\alpha$ being irrational. The analysis is essentially the same as that for the Carleman model. However, the modified particle method approximation to the original Broadwell model with $\alpha=0$ is more subtle. The low order interpolation scheme using a discrete convolution would not be accurate enough to capture the resonant interaction among the three components of the solution. On the other hand, we found convincing numerical evidences which indicate that high order interpolation schemes such as cubic spline or spectral interpolations capture the resonant interaction of the solution very well even on a coarse grid. We present some numerical results of this study in the next section.

The reason why the convergence proof can be extended to the Broadwell model in the non-resonant case is because only the averages of $u$ and $v$ would affect the third component $w$, as revealed by the homogenization result given by (8). Thus the interaction among the three components of the solution is not sensitive to the interpolation scheme being used.

\section{Numerical results.}

5.1. Numerical results for $1 D$ Carleman and Broadwell equations. In this subsection, we perform two sets of numerical experiments to illustrate the convergence of our modified particle method. In the first experiment, we consider the modified particle algorithm (12), (14) applied to the variable coefficients Carleman model (2). The convergence of this scheme was proved in Section 4 in the case when the interpolation is done by using discrete convolutions. The second set of numerical experiments considers the analogue of the scheme (12)-(14) for the Broadwell model (4). This algorithm is given by (60) and (61).

Carleman model. We consider the following initial data for the variable coefficient Carleman model:

$$
u(x, 0)= \begin{cases}0.5 \sin ^{4}\left(\frac{\pi(x-3)}{2}\right)\left(1+\sin \left(\frac{2 \pi(x-3)}{\epsilon}\right)\right) & |x-4|<1 \\ 0 & |x-4| \geq 1\end{cases}
$$

and

$$
v(x, 0)= \begin{cases}0.5 \sin ^{4}\left(\frac{\pi(x-4)}{2}\right)\left(1+\sin \left(\frac{2 \pi(x-4)}{\epsilon}\right)\right) & |x-5|<1 \\ 0 & |x-5| \geq 1\end{cases}
$$




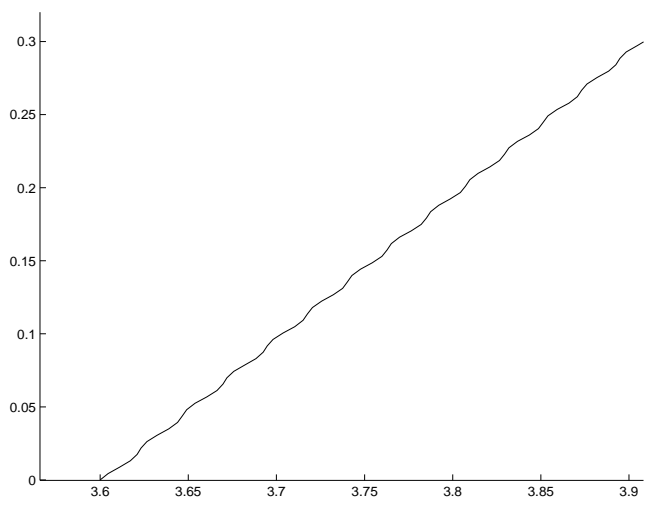

(a)

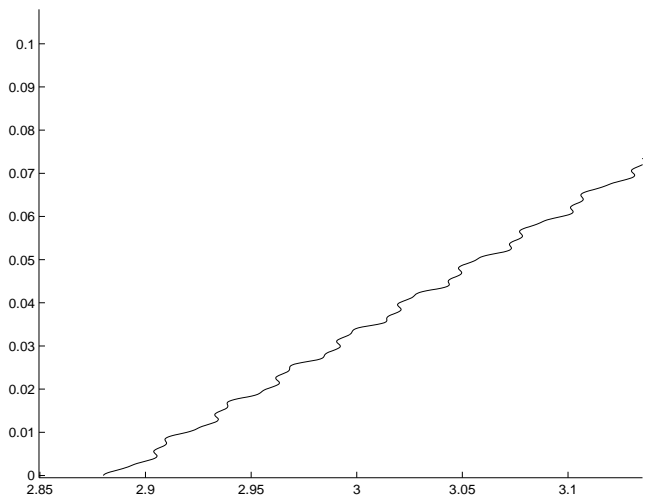

(b)

FIG. 2. Typical characteristic lines corresponding to the oscillatory velocity field $a(x, t)$ given by (57a) and (59a), respectively. Note that the exact characteristics are oscillatory.

where $\epsilon=\sqrt{2} / 100 \approx 0.014$. This particular initial condition was used in [6].

To make a more severe test, we consider oscillatory variable coefficients:

$$
\begin{aligned}
& a(x, t)=1+0.5 \sin \left(\frac{x t}{\epsilon}\right), \\
& b(x, t)=1+0.2 \cos \left(\frac{x t}{\epsilon}\right) .
\end{aligned}
$$

A typical characteristic line is plotted in Figure 2(a). Note that the exact characteristics are oscillatory.

We run the modified Lagrangian algorithm (12)-(14) with a coarse space and time mesh. We take the spatial gridsize to be $\Delta x=0.01$ and the timestep $\Delta t=\frac{\Delta x}{\sqrt{5}}$. Thus $\Delta x$ and $\Delta t$ are of order $O(\epsilon)$. The numerical solution obtained with the coarse mesh is compared to an "accurate" solution obtained by using a very fine grid with $\Delta x=0.001$ and $\Delta t=0.00025$. Note that $\Delta x$ is related to $\epsilon$ by $\epsilon=\Delta x \sqrt{2}$. We make this choice of the coarse grid mesh to avoid the resonant sampling error between the coarse gridsize and the oscillations (see (21)). We use a discrete convolution-based interpolation with the cut-off function given by the "cosine function" (16). The width of the cut-off function is taken to be $\sigma=\Delta x^{\beta}$, with $\beta=0.75$. 


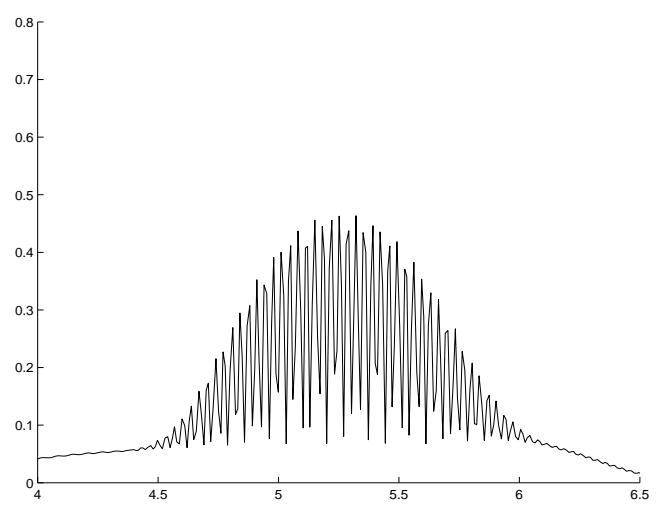

FIG. 3. Solution $u$ at time $t=1.28$ for the oscillatory coefficient Carleman model $(\epsilon \approx 0.014)$ using the algorithm given by (12) and (14) with $\Delta x=0.01$. Note that the coarse grid solution preserves the highly oscillatory features.

Discrete averages. One of the main issues in designing good methods for oscillatory problems is to obtain correct averaged solutions. The formula for the dynamical average that we use is given by discrete convolutions as in [6]. The discrete average for the numerical solution $u_{i}^{n}$ is defined by

$$
\overline{u_{i}^{n}}=\Delta x \sum_{k} \theta_{\sigma}\left(x_{i}-\tilde{x}_{u, k}^{n}\right) u_{k}^{n} \frac{\partial \Phi}{\partial x}\left(t_{n} ; 0, x_{k}\right),
$$

where $\tilde{x}_{u, k}^{n}$ represent the numerical $u$ characteristics and $\theta_{\sigma}$ is a cut-off function of width $\sigma$, constructed by (15) using the cosine shape function (16) and $\sigma=\Delta x^{\beta}$ with $\beta=0.4$. The values $\frac{\partial \Phi}{\partial x}\left(t_{n} ; 0, x_{k}\right)$ are computed numerically by discretizing:

$$
\frac{d}{d t}\left(\frac{\partial \Phi}{\partial x}\right)=\frac{\partial a}{\partial x} \frac{\partial \Phi}{\partial x},\left.\quad \frac{\partial \Phi}{\partial x}\right|_{t=0}=1 .
$$

In Figure 3 we plot the numerical solution $u$ at $t=1.28$ obtained with the modified particle algorithm (12), (14), using a coarse mesh. We observe that the oscillatory features are well captured. Moreover, in Figure 4 we plot the solution of the coarse grid computation on top of a well-resolved computation. We include only a portion of this plot in order to see how well the coarse grid computation (the circles) recovers the main features (mean, amplitude of oscillations) of the oscillatory pulse (solid line).

Figure 5 displays the moving averages (see equation (58)) of the numerical solution $u$ computed using a coarse and a fine grid respectively. We observe that the average of the coarse grid solution (the dash-dot line) agrees very well with that of the fine grid solution, even in the oscillatory region. An excellent agreement is also obtained for the averaged second order moment $\overline{u^{2}}$ (see Figure 6). The latter test is an indication of how well the amplitudes of the oscillations are recovered.

Interpolation schemes. The above numerical results, which are obtained using discrete convolution as interpolation, support the convergence analysis in Section 4. In fact, we consider a more difficult problem, by allowing the coefficients $a(x, t)$ and $b(x, t)$ to be oscillatory.

We have also considered other interpolation schemes such as cubic spline and linear interpolation schemes for the modified particle method (12),(14). They all produce results very similar to the ones depicted in Figures 3-6. 


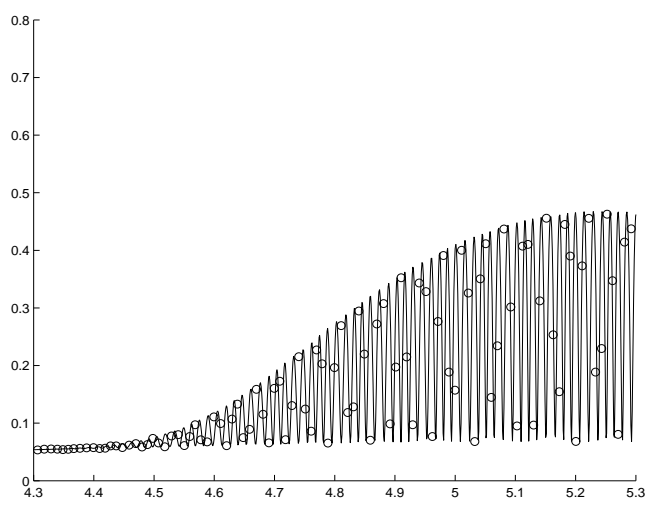

Fig. 4. Numerical solution $u$ at time $t=1.28$ for the oscillatory coefficient Carleman model with $\epsilon \approx 0.014$. The o's represent the coarse grid with $\Delta x=0.01$. The solid line represents a very well resolved computation with $\Delta x=0.001$.

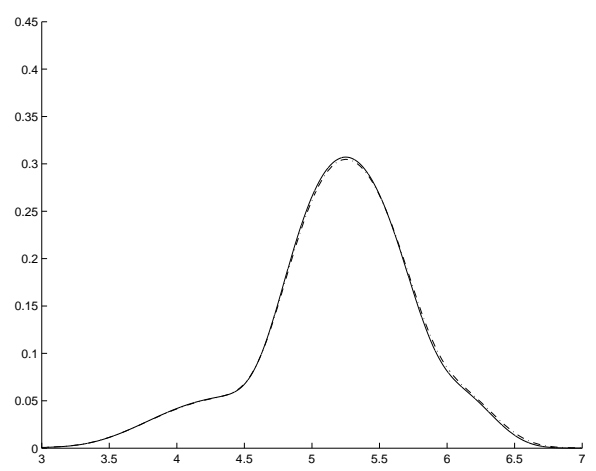

FIG. 5. Averaged solution $\bar{u}$ (dash-dot line) at time $t=1.28$ for the oscillatory coefficient Carleman model $(\epsilon \approx 0.014)$ with a coarse mesh $\Delta x=0.01$. Note the excellent agreement with a very well resolved computation (solid line) with $\Delta x=0.001$.

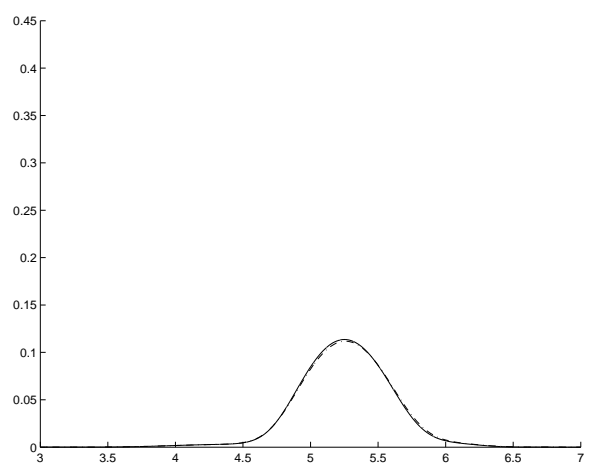

FIG. 6. Averaged second order moment $\overline{u^{2}}$ (dash-dot line) at time $t=1.28$ for the oscillatory coefficient Carleman model $(\epsilon \approx$ 0.014) with a coarse mesh $\Delta x=0.01$. The solid line represents a very well resolved computation with $\Delta x=0.001$.

Numerical convergence. Table 1 contains a list of numerical errors in the averaged solution $\bar{u}$ of the oscillatory coefficient Carleman model using the algorithm (12), (14). Here, $\epsilon \approx 0.014$ and the "accurate" solution is computed with $\Delta x=0.001$. The convergence is better than first order accuracy in $l^{1}, l^{2}$ and the maximum norms.

A case with highly oscillatory velocity coefficients. We investigate to a certain extent how the accuracy of the modified particle method changes when the coefficients $a(x, t), b(x, t)$ become highly oscillatory. Consider the Carleman equations with the same initial conditions, given by (55), (56), and with the variable coefficients $a(x, t)$ and $b(x, t)$ given by

$$
\begin{aligned}
& a(x, t)=1+0.75\left(\sin \left(\frac{2 \pi t}{\epsilon}\right)+\sin \left(\frac{\pi x}{\epsilon}\right)\right), \\
& b(x, t)=1+0.7\left(\sin \left(\frac{2 \pi t}{\epsilon}\right)+\cos \left(\frac{\pi x}{\epsilon}\right)\right) .
\end{aligned}
$$


TABLE 1

Errors in the solution $\bar{u}$ of the oscillatory coefficient Carleman model for the algorithm (12), (14). Here, $\epsilon \approx 0.014$ and the "accurate" solution is computed with $\Delta x=0.001$.

\begin{tabular}{|r|r|r|r|}
\hline Gridsize & $l^{1}$-norm & $l^{2}$-norm & Max-norm \\
\hline 0.02 & 0.0328 & 0.0236 & 0.0326 \\
0.01 & 0.0061 & 0.0042 & 0.0048 \\
0.005 & 0.0024 & 0.0016 & 0.0018 \\
0.002 & $7.2 \times 10^{-4}$ & $4.9 \times 10^{-4}$ & $5.7 \times 10^{-4}$ \\
\hline
\end{tabular}

As before, $\epsilon=\sqrt{2} / 100 \approx 0.014$.

A typical characteristic line is plotted in Figure 2(b). Note that the exact characteristics are more oscillatory than those presented in Figure 2(a) that corresponds to $(57 \mathrm{a}))$.

A coarse space and time computation $\left(\Delta x=0.01, \Delta t=\frac{\Delta x}{\sqrt{5}}\right)$ using the modified particle method leads to relatively unsatisfactory results in terms of obtaining correct averaged solutions. However, by taking a smaller timestep $(\Delta t=0.001)$, the results improve greatly. We do not present the results here, as they are very similar to the results presented above (see Figures 5-6 or Table 1). We conjecture that this behavior is generic, namely a coarse Lagrangian method may work for problems involving highly oscillatory characteristics (particle paths) if the characteristics are well resolved in time.

Broadwell model. We now study numerically the analogue of the algorithm (12), (14) for the Broadwell system (6). In (6), the characteristics are known exactly. Hence, the discrete characteristics are updated by

$$
\begin{aligned}
& x_{i, u}^{n+1}=x_{i, u}^{n}+\Delta t, \\
& x_{i, v}^{n+1}=x_{i, v}^{n}-\Delta t, \\
& x_{i, w}^{n+1}=x_{i, w}^{n}+\alpha \Delta t .
\end{aligned}
$$

Let $u_{i}^{n}, v_{i}^{n}$ and $w_{i}^{n}$ denote the numerical approximations of $u\left(x_{i, u}^{n}, t_{n}\right), v\left(x_{i, v}^{n}, t_{n}\right)$ and $w\left(x_{i, w}^{n}, t_{n}\right)$, respectively. The modified particle scheme for the Broadwell system is given by

$$
\begin{aligned}
u_{i}^{n+1} & =u_{i}^{n}+\Delta t\left(\mathcal{I}_{n}\left(w^{n}\right)^{2}{ }_{i}-u_{i}^{n} \mathcal{I}_{n} v_{i}^{n}\right) \\
v_{i}^{n+1} & =v_{i}^{n}+\Delta t\left(\mathcal{I}_{n}\left(w^{n}\right)^{2}{ }_{i}-v_{i}^{n} \mathcal{I}_{n} u_{i}^{n}\right) \\
w_{i}^{n+1} & =w_{i}^{n}-\Delta t\left(\left(w_{i}^{n}\right)^{2}-\left(\mathcal{I}_{n} u_{i}^{n}\right) \cdot\left(\mathcal{I}_{n} v_{i}^{n}\right)\right) .
\end{aligned}
$$

In (61), (a)-(c), $\mathcal{I}_{n}$ denotes interpolation at the points $x_{i, u}^{n}, x_{i, v}^{n}$ and $x_{i, w}^{n}$, respectively.

In the numerical experiments we take the initial data for $u$ and $v$ as in (55) and (56) and zero initial values for $w$. We take $\epsilon \approx 0.0138$ and compute the solution using the algorithm (60)-(61) with a coarse space and time mesh, $\Delta x=0.01$ and $\Delta t=\frac{\Delta x}{\sqrt{5}}$. We note again that $\Delta x$ and $\Delta t$ are of order $O(\epsilon)$. We compare the coarse grid solution with an "accurate" solution obtained by using a very fine grid with $\Delta x=1.25 \times 10^{-3}$.

In view of the homogenization results for (6) (see equations (7) and (8)), we consider two cases:

Case I: $\alpha$ is rational (the resonance case). We take $\alpha=0$, so (6) reduces to (4). 


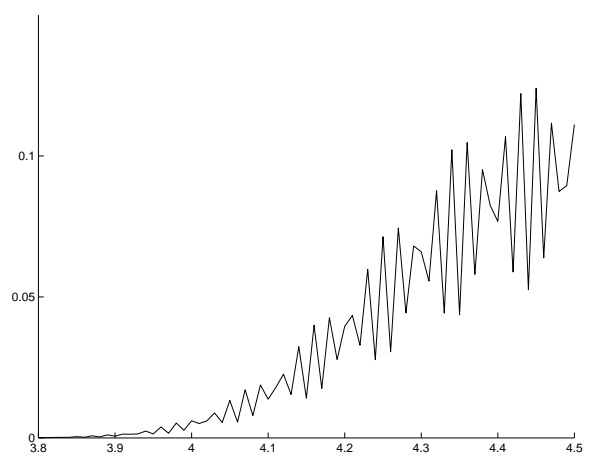

FIG. 7. Solution $w$ at time $t=2.5$ for the Broadwell model $(\epsilon \approx 0.0138)$ with $\alpha=0$ using the modified particle scheme (60)-(61) with $\Delta x=0.01$.

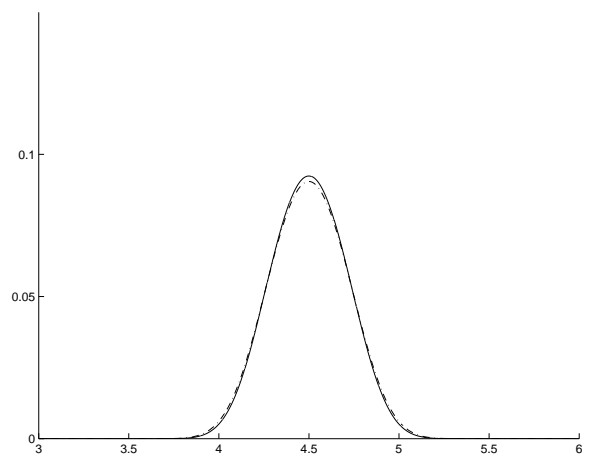

FIG. 9. Averaged solution $\bar{w}$ at time $t=$ 2.5 for the Broadwell model (4) using a coarse grid $\Delta x=0.01$ (dash-dot line), and a fine grid $\Delta x=1.25 \times 10^{-3}$ (solid line).

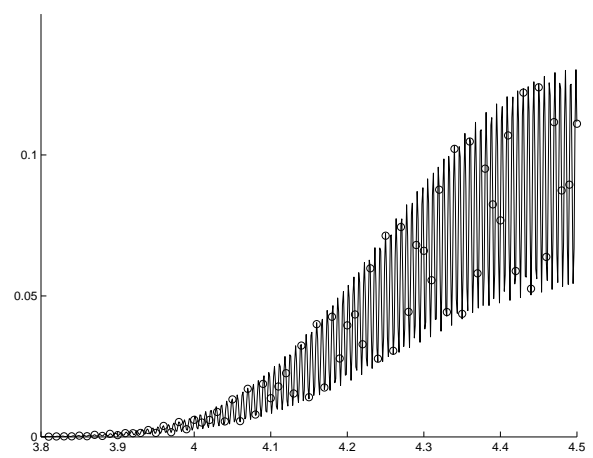

FIG. 8. Numerical solution $w$ at time $t=2.5$ for the Broadwell model (4) $(\epsilon \approx$ 0.0138 ), $\Delta x=0.01$ (circles) and $\Delta x=$ $1.25 \times 10^{-3}$ (solid line).

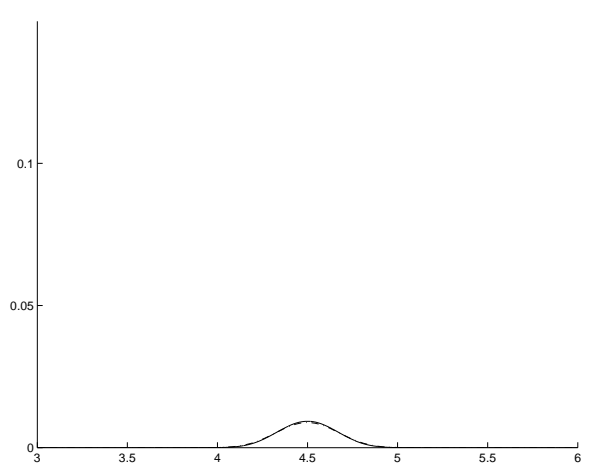

Fig. 10. Averaged second order moment $\overline{w^{2}}$ at time $t=2.5$ for the Broadwell model (4) using a coarse grid $\Delta x=0.01$ (dash-dot line), and a fine grid $\Delta x=1.25 \times 10^{-3}$ (solid line).

In Figure 7 we plot the numerical solution $w$ at $t=2.5$ obtained by the modified particle scheme (60)-(61) with a coarse grid. As we know from the homogenization equations, the nonlinear interaction of the $u v$ term in (4) generates an oscillatory pulse on $w$. We observe in Figure 7 that the oscillatory features are well captured by the modified particle method on a coarse grid. In Figure 8 we plot the solution of the modified particle method using a coarse grid on top of a well-resolved computation. We note that the coarse grid computation (the circles) recovers very well the main features (mean, amplitude of oscillations) of the oscillatory pulse (solid line).

In Figures 9 and 8 we test how well the coarse modified particle method captures the mean and the second order moment of the solution. We find excellent agreement with the results of a fine grid computation.

Interpolation schemes. We experiment with various interpolation schemes in algorithm (60)-(61). The results presented in Figures 7-10 are obtained by using a cubic spline interpolation. We believe that higher order interpolation schemes (cubic spline or Fourier) are important to capture the resonant interaction of the three components of the solution. Our numerical experiments show that the modified particle method 


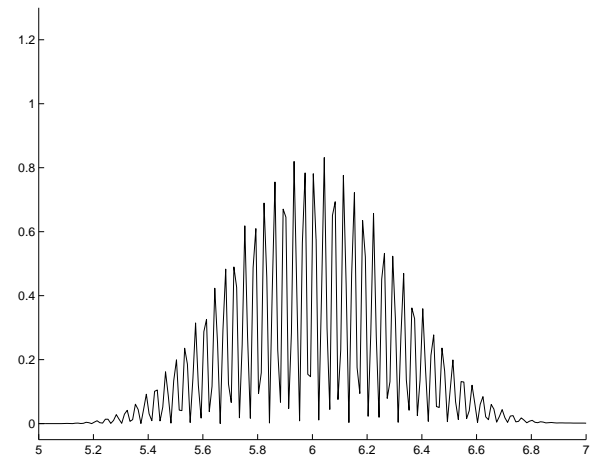

FIG. 11. Solution $u$ at time $t=2.0$ for the Broadwell model (6) with $\alpha=\pi / 2$ with $\Delta x=0.01$.

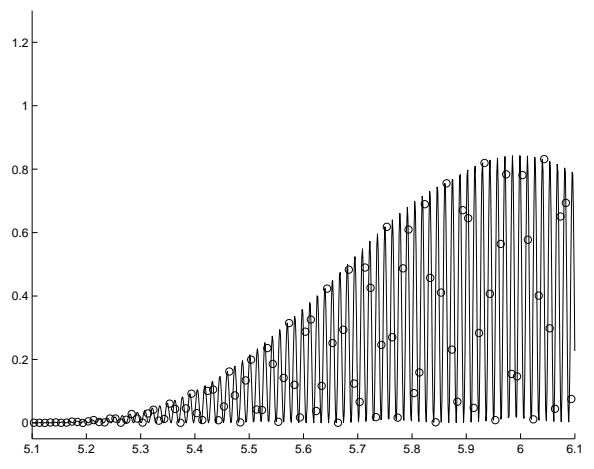

FIG. 12. Solution $u$ at time $t=2.0$ for the Broadwell model (6) with $\alpha=\pi / 2, \Delta x=$ 0.01 (circles) and $\Delta x=1.25 \times 10^{-3}$ (solid line).

with a cubic spline interpolation captures accurately the mean and the amplitude of an oscillating pulse, even if we integrate the solution for a large number of time steps. On the other hand, our experiments indicate that the modified particle method using a linear or discrete convolution interpolation scheme with $\sigma \gg \Delta x$ would not capture the oscillatory features of $w$.

Numerical convergence. Table 2 contains the numerical errors in the averaged solution $\bar{w}$ of the Broadwell model (4) for algorithm (60)-(61). Here, $\epsilon \approx 0.0138$ and the "accurate" solution is computed with $\Delta x=1.25 \times 10^{-3}$. We observe that the averaged solution converges with first order accuracy.

TABLE 2

Errors in the solution $\bar{w}$ of the Broadwell model with $\alpha=0$ for the algorithm (60)-(61). Here, $\epsilon \approx 0.0138$ and the "accurate" solution is computed with $\Delta x=1.25 \times 10^{-3}$.

\begin{tabular}{|r|c|c|c|}
\hline Gridsize & $l^{1}$-norm & $l^{2}$-norm & Max-norm \\
\hline 0.02 & 0.0022 & 0.0021 & 0.0030 \\
0.01 & 0.0013 & 0.0013 & 0.0019 \\
0.005 & $4.9 \times 10^{-4}$ & $4.7 \times 10^{-4}$ & $6.5 \times 10^{-4}$ \\
0.0025 & $2.3 \times 10^{-4}$ & $2.2 \times 10^{-4}$ & $3.4 \times 10^{-4}$ \\
\hline
\end{tabular}

Case II: $\alpha=$ irrational (the non-resonance case). We take $\alpha=\pi / 2$ in (6).

In this case, the homogenization result indicates that non-oscillatory initial data for $w$ will remain non-oscillatory at later times. This is because the $u$ and $v$ components enter the homogenized $w$-equation only through their averaged values. On the other hand, oscillatory initial data for $u$ and $v$ will stay oscillatory at later times. This subtle aspect is well captured by the modified particle method on a coarse grid.

Figure 11 displays the solution $u$ of the coarse grid computation at time $t=2.0$. We notice that the oscillatory features are captured. In Figure 12 we plot the coarse grid solution $u$ on top of a very well resolved solution. We notice that the mean and the amplitude of the oscillatory pulse are very well preserved by the coarse grid solution.

In Figure 13 we plot the mean of the solution $u$ obtained by the coarse grid 


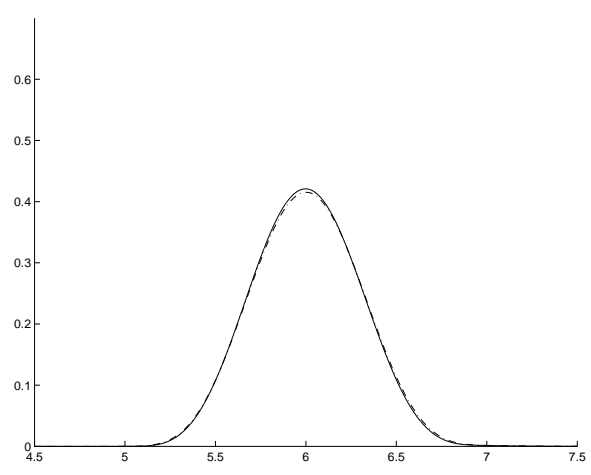

FIG. 13. Averaged solution $u$ at time $t=$ 2.0 for the Broadwell model (6) with $\alpha=\pi / 2$, $\Delta x=0.01$ (dash-dot line) and $\Delta x=1.25 \times$ $10^{-3}$ (solid line).

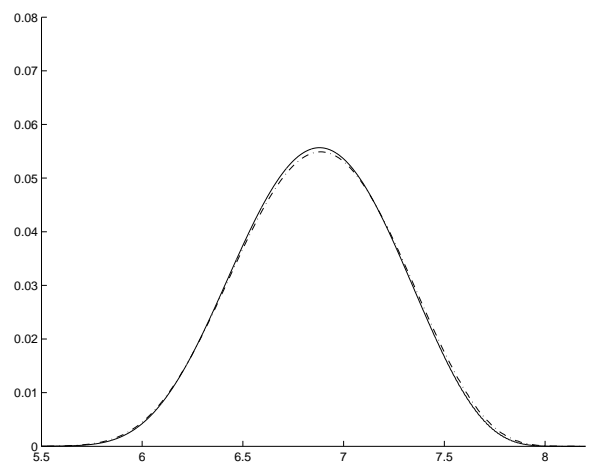

FIG. 14. Averaged solution $w$ at time $t=2.0$ for the Broadwell model (6) with $\alpha=$ $\pi / 2, \Delta x=0.01$ (dash-dot line) and $\Delta x=$ $1.25 \times 10^{-3}$ (solid line).

computation and compare it with a very-well resolved computation. We again see excellent agreement between the coarse and the fine grid computations. Figure 14 displays the solution $w$ of the coarse grid computation at time $t=2.0$ together with the solution of a very well-resolved computation. The agreement between the two numerical solutions is excellent.

Interpolation schemes. We also experiment with various interpolation schemes in algorithm (60), (61) for this non-resonant case. The results presented in Figures 11-14 are obtained by using a discrete convolution-based interpolation with the cut-off function given by (16). The width of the cut-off function was taken to be $\sigma=\Delta x^{\beta}$, with $\beta=0.75$. Unlike the resonant case, lower order interpolation schemes (linear interpolation, discrete convolutions with $\sigma \gg \Delta x$ ) works equally well as the high order interpolation schemes. The oscillatory features of the solution is well preserved. This is due to the fact that there is no resonant interaction in the $w$ equation. Only the averages of $u$ and $v$ enter to the homogenized $w$-equation. Thus the interaction is not sensitive to the interpolation scheme being used.

Numerical convergence. We display in Table 3 the numerical errors in the averaged solution $\bar{u}$ of the Broadwell model (6) with $\alpha=\pi / 2$ for algorithm (60)-(61). Here, $\epsilon \approx$ 0.0138 and the "accurate" solution is computed with $\Delta x=1.25 \times 10^{-3}$. Convergence in all three norms is evident.

TABLE 3

Errors in the solution $\bar{u}$ of the Broadwell model with $\alpha=\pi / 2$ for the algorithm (60), (61). Here, $\epsilon \approx 0.0138$ and the "accurate" solution is computed with $\Delta x=1.25 \times 10^{-3}$.

\begin{tabular}{|r|c|c|c|}
\hline Gridsize & $l^{1}$-norm & $l^{2}$-norm & Max-norm \\
\hline 0.02 & 0.0090 & 0.0077 & 0.0109 \\
0.01 & 0.0052 & 0.0046 & 0.0063 \\
0.005 & 0.0023 & 0.0020 & 0.0028 \\
0.0025 & $7.8 \times 10^{-4}$ & $6.7 \times 10^{-4}$ & $9.7 \times 10^{-4}$ \\
\hline
\end{tabular}

5.2. Numerical results for $2 D$ Carleman equations. In this subsection we investigate the performance of the modified particle method for the $2 D$ Carleman 
equations with constant coefficients, given by

$$
\begin{aligned}
& \frac{\partial u}{\partial t}+a_{1} \frac{\partial u}{\partial x}+a_{2} \frac{\partial u}{\partial y}+u^{2}-v^{2}=0 \\
& \frac{\partial v}{\partial t}+b_{1} \frac{\partial v}{\partial x}+b_{2} \frac{\partial v}{\partial y}-u^{2}+v^{2}=0
\end{aligned}
$$

where $a_{1}, a_{2}, b_{1}, b_{2}$ are constants.

The numerical results presented in this subsection correspond to the velocity fields:

$$
\left(a_{1}, a_{2}\right)=(1,1) \quad \text { and } \quad\left(b_{1}, b_{2}\right)=(-1,1) .
$$

We consider initial data with multiple scales. In addition to periodic initial data (a $2 D$ analogue of (3)), we also consider initial data with random phase.

Periodic initial data. We consider the following periodic initial data:

$$
u(x, y, 0)=\left\{\begin{array}{c}
0.5 \sin ^{3}\left(\frac{\pi(x-2)}{2}\right) \sin ^{3}\left(\frac{\pi(y-3)}{2}\right)\left(1+0.5 \sin \left(\frac{2 \pi(x-2)}{\epsilon}\right)\right. \\
\left.\quad+0.5 \sin \left(\frac{2 \pi(y-3)}{\epsilon}\right)\right), \quad \text { for }|x-3|<1 \text { and }|y-4|<1 \\
0 \quad \text { otherwise, }
\end{array}\right.
$$

and

$$
v(x, y, 0)=\left\{\begin{array}{c}
0.5 \sin ^{3}\left(\frac{\pi(x-3)}{2}\right) \sin ^{3}\left(\frac{\pi(y-3)}{2}\right)\left(1+0.5 \sin \left(\frac{2 \pi(x-3)}{\epsilon}\right)\right. \\
\left.\quad+0.5 \sin \left(\frac{2 \pi(y-3)}{\epsilon}\right)\right), \quad \text { for }|x-4|<1 \text { and }|y-4|<1 \\
0 \quad \text { otherwise } .
\end{array}\right.
$$

Here, $\epsilon=0.07 \sqrt{2} \approx 0.0989$.

We run the coarse modified particle method with $\Delta x=8 / 128=0.0625$ and $\Delta t=\Delta x / \sqrt{5}$. This space meshsize corresponds to $N=128^{2}$ equally spaced particles in a square of size 8 . We compare the results with a very well resolved computation with $\Delta x=8 / 1024 \approx 0.0078$ and $\Delta t=\Delta x / 2$. For the well resolved computation we use pseudospectral techniques and update the solution in the Fourier space. The integration in time is done using the fourth-order Runge-Kutta method.

The results are presented in Figures 15-17. The figures represent cross sections of the numerical solution $u$ at time $t=1.25$. They show an excellent agreement of the coarse modified particle method with the well resolved computation. The averages in Figures 16-17 are computed by discrete convolutions (the $2 D$ analogue of (58)) with the cut-off function

$$
\theta_{\sigma}(x, y)= \begin{cases}1 / \sigma^{2} & (x, y) \in[-\sigma, \sigma] \times[-\sigma, \sigma] \\ 0 & \text { otherwise }\end{cases}
$$

where $\sigma=0.35$.

Table 4 provides numerical errors that indicate the excellent performance of the coarse particle method in the $2 D$ case. The relative $l^{2}$ - and maximum-norm errors of the coarse particle method solution are computed with respect to the "accurate" solution, obtained by a very well-resolved computation with $\Delta x \approx 0.0078$. 
TABLE 4

Coarse modified particle method $(\Delta x=0.0625)$ : relative errors in the solution $\bar{u}$ (first row) and $\overline{u^{2}}$ (second row). The "accurate" solution is obtained by a very well resolved computation with $\Delta x \approx 0.0078$. Here, $\epsilon \approx 0.098$.

\begin{tabular}{|c|c|c|}
\hline & relative $l^{2}$-norm & relative max-norm \\
\hline error in $\bar{u}$ & 0.0140 & 0.0276 \\
error in $\overline{u^{2}}$ & 0.0175 & 0.0363 \\
\hline
\end{tabular}

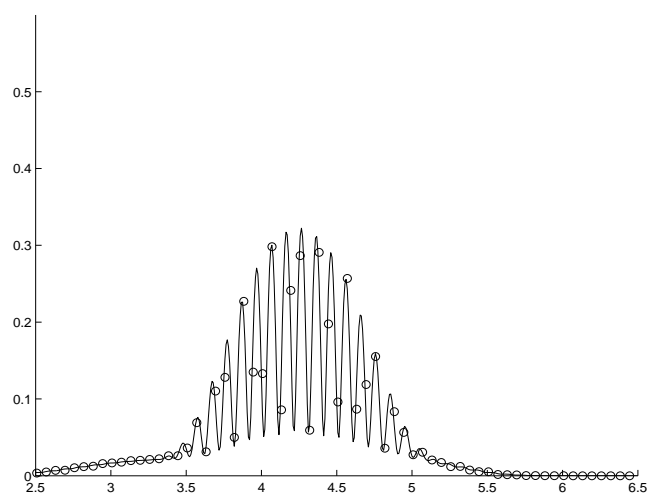

FIG. 15. Cross section ( $y=$ const.) of the solution $u$ at time $t=1.25$ for the $2 D$ constant coefficient Carleman model with periodic initial data $(\epsilon \approx 0.098)$. The o's represent the coarse grid $(\Delta x=0.0625)$ computation using the modified particle algorithm. The solid line represents a very well resolved computation with $\Delta x \approx 0.0078$.

Random initial data. We performe a more challenging test for an initial condition similar to (63), (64), but with a random phase, i.e.

$u(x, y, 0)=\left\{\begin{array}{l}0.5 \sin ^{3}\left(\frac{\pi(x-2)}{2}\right) \sin ^{3}\left(\frac{\pi(y-3)}{2}\right)\left[1+0.5 \sin \left(2 \pi\left(\frac{x-2}{\epsilon}+\theta_{1}(x, y)\right)\right)\right. \\ \left.\quad+0.5 \sin \left(2 \pi\left(\frac{y-3}{\epsilon}+\theta_{1}(x, y)\right)\right)\right], \quad \text { for }|x-3|<1 \text { and }|y-4|<1 \\ 0 \quad \text { otherwise },\end{array}\right.$

and

$$
v(x, y, 0)=\left\{\begin{array}{c}
0.5 \sin ^{3}\left(\frac{\pi(x-3)}{2}\right) \sin ^{3}\left(\frac{\pi(y-3)}{2}\right)\left[1+0.5 \sin \left(2 \pi\left(\frac{x-3}{\epsilon}+\theta_{2}(x, y)\right)\right)\right. \\
\left.\quad+0.5 \sin \left(2 \pi\left(\frac{y-3}{\epsilon}+\theta_{2}(x, y)\right)\right)\right], \quad \text { for }|x-4|<1 \text { and }|y-4|<1 \\
0 \quad \text { otherwise }
\end{array}\right.
$$

Here, $\theta_{1}(x, y)$ and $\theta_{2}(x, y)$ are random variables that take values in the interval $[0,1]$ and $\epsilon=0.07 \sqrt{2} \approx 0.0989$. To initialize our code with the initial data (66), (67), we use a random number generator to get the values $\theta_{1}\left(x_{i}, y_{j}\right), \theta_{2}\left(x_{i}, y_{j}\right)$ at each grid point $\left(x_{i}, y_{j}\right)$. We generate the initial data by evaluating (66), (67) on an equally spaced $256 \times 256$ grid in a square of size $8(\Delta x=8 / 256 \approx 0.0312)$. Then we interpolate these values on a $1024 \times 1024$ grid. This will ensure that the initial random oscillations are well resolved. 


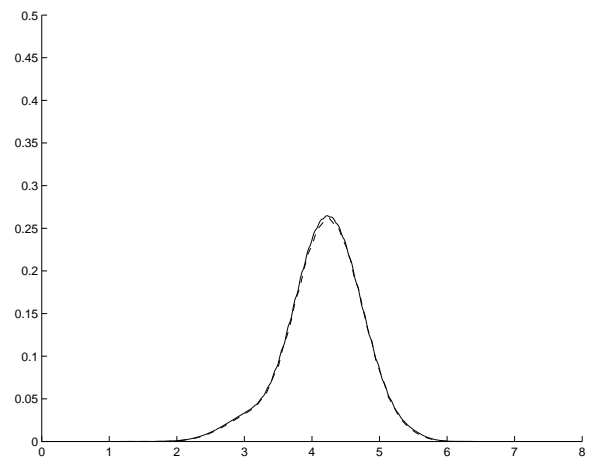

FIG. 16. Cross section ( $y=$ const.) of the averaged solution $\bar{u}$ at time $t=1.25$ for the $2 D$ constant coefficient Carleman model with periodic initial data $(\epsilon \approx 0.098)$. The dash-dot line corresponds to a computation using the modified particle method with a coarse mesh, $\Delta x=0.0625$. Note the excellent agreement with a well resolved computation with $\Delta x \approx 0.0078$ (solid line).

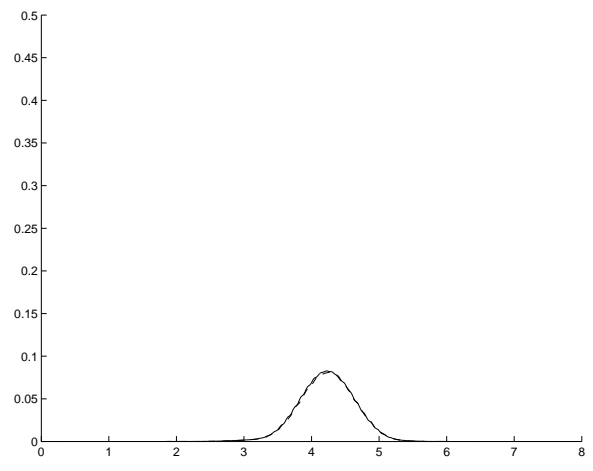

FIG. 17. Cross section ( $y=$ const.) of the averaged second order moment $\overline{u^{2}}$ at time $t=1.25$ for the $2 D$ constant coefficient Carleman model with periodic initial data $(\epsilon \approx 0.098)$. The dash-dot line corresponds to a computation using the modified particle method with a coarse mesh, $\Delta x=0.0625$. The solid line represents a well resolved computation with $\Delta x \approx 0.0078$.

We perform a similar comparison as the one presented in the previous paragraph. The coarse modified particle method is run with $N=128^{2}$ particles $(\Delta x=0.0625)$. The well-resolved computation uses a pseudospectral code on a $1024^{2}$ grid $(\Delta x \approx$ 0.0078 ). The averages are computed using discrete convolutions with $\theta_{\sigma}$ given by $(65)$ with $\sigma=0.35$. The results are presented in Figures 18-20.

Table 5 displays the numerical relative $l^{2}$ - and maximum-norm errors of the coarse particle method solution for initial data with random phase. The errors are computed with respect to the "accurate" solution, obtained by a very well-resolved computation with $\Delta x \approx 0.0078$.

TABLE 5

Initial data with random phase: relative errors in the solution $\bar{u}$ (first row) and $\overline{u^{2}}$ (second row) computed with a coarse modified particle method $(\Delta x=0.0625)$. The "accurate" solution is obtained by a very well resolved computation with $\Delta x \approx 0.0078$. Here, $\epsilon \approx 0.098$.

\begin{tabular}{|c|c|c|}
\hline & relative $l^{2}$-norm & relative max-norm \\
\hline error in $\bar{u}$ & 0.0202 & 0.0359 \\
error in $\overline{u^{2}}$ & 0.0342 & 0.0701 \\
\hline
\end{tabular}

The numerical results for the multi-dimensional Carleman model with random initial data are very encouraging. The modified particle method presented in this paper can be applied to more general semi-linear hyperbolic systems with random initial data. In the future, we would like to investigate how to extend this method to more general multi-dimensional convection dominated transport problems. 


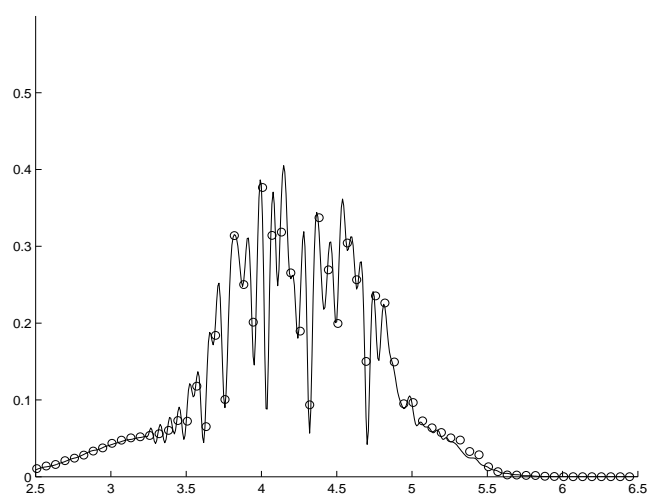

FIG. 18. Cross section ( $y=$ const.) of the solution $u$ at time $t=1.25$ for the $2 D$ constant coefficient Carleman model with random initial data. The o's represent the coarse grid $(\Delta x=$ $0.0625)$ computation using the modified particle algorithm. The solid line represents a very well resolved computation with $\Delta x \approx 0.0078$.

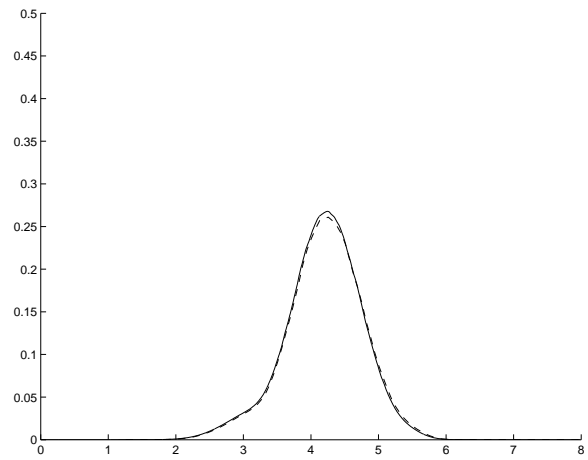

FIG. 19. Cross section ( $y=$ const.) of the averaged solution $\bar{u}$ at time $t=1.25$ for the $2 D$ constant coefficient Carleman model with random initial data. The dash-dot line corresponds to a computation using the modified particle method with a coarse mesh, $\Delta x=0.0625$. Note the excellent agreement with a well resolved computation with $\Delta x \approx 0.0078$ (solid line).

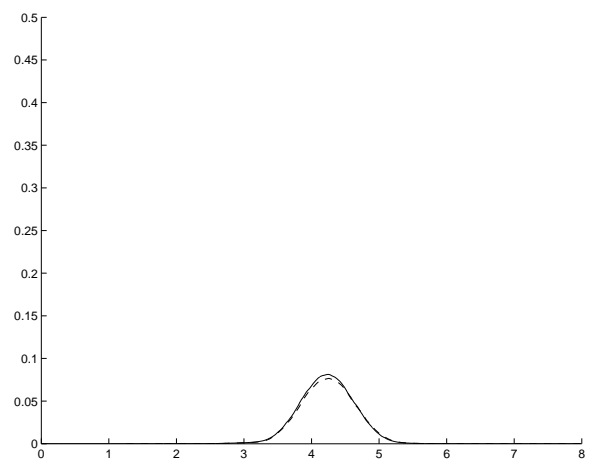

FiG. 20. Cross section ( $y=$ const.) of the averaged second order moment $\overline{u^{2}}$ at time $t=1.25$ for the $2 D$ constant coefficient Carleman model with random initial data. The dash-dot line corresponds to a computation using the modified particle method with a coarse mesh, $\Delta x=0.0625$. The solid line represents a well resolved computation with $\Delta x \approx 0.0078$.

\section{Appendix.}

\subsection{Proof of Lemma 4.5.}

Proof. We prove the lemma by induction. Suppose that at the discrete time $t_{n}$ we have

$$
0 \leq u_{i}^{n}, v_{i}^{n} \leq M_{n}, \quad i \in \mathbb{Z},
$$

where $M_{n}$ is a constant. Note that

$$
\frac{\partial \Psi}{\partial x}(s ; t, x)=\exp \left(\int_{t}^{s}-\frac{\partial b}{\partial x}(\Psi(\tau ; t, x), \tau) d \tau\right)>0 .
$$


Then, we obtain

$$
\begin{aligned}
\mathcal{I}_{n}\left(v^{n}\right)_{i}^{2}= & \Delta x \sum_{j} \theta_{\sigma}\left(x_{i, u}^{n}-x_{j, v}^{n}\right)\left(v_{j}^{n}\right)^{2} \frac{\partial \Psi}{\partial x}\left(t_{n} ; 0, x_{j}\right) \\
\leq & M_{n}^{2} \Delta x \sum_{j} \theta_{\sigma}\left(x_{i, u}^{n}-x_{j, v}^{n}\right) \frac{\partial \Psi}{\partial x}\left(t_{n} ; 0, x_{j}\right) \\
= & M_{n}^{2}\left[\Delta x \sum_{j} \theta_{\sigma}\left(\Phi\left(t_{n} ; 0, x_{i}\right)-\Psi\left(t_{n} ; 0, x_{j}\right)\right) \frac{\partial \Psi}{\partial x}\left(t_{n} ; 0, x_{j}\right)\right. \\
& \left.-\int_{\mathbb{R}} \theta_{\sigma}\left(\Phi\left(t_{n} ; 0, x_{i}\right)-\Psi\left(t_{n} ; 0, y\right)\right) \frac{\partial \Psi}{\partial y}\left(t_{n} ; 0, y\right) d y+1\right]
\end{aligned}
$$

where we have used the fact that $\int \theta_{\sigma}(x-y) d y=1$ and $\theta_{\sigma} \geq 0$. Due to the finite support of $\theta_{\sigma}$, the nonzero contribution to the integral over $y$ in the RHS of the above equation comes from the interval defined by

$$
\left|\Phi\left(t_{n} ; 0, x_{i}\right)-\Psi\left(t_{n} ; 0, y\right)\right| \leq \sigma .
$$

The length of such an interval is of order $O(\sigma)$. Hence, using the error estimate for the rectangle rule approximation [10], we obtain

$$
\begin{aligned}
& \mid \Delta x \sum_{j} \theta_{\sigma}\left(\Phi\left(t_{n} ; 0, x_{i}\right)-\Psi\left(t_{n} ; 0, x_{j}\right)\right) \frac{\partial \Psi}{\partial x}\left(t_{n} ; 0, x_{j}\right) \\
& -\int_{\mathbb{R}} \theta_{\sigma}\left(\Phi\left(t_{n} ; 0, x_{i}\right)-\Psi\left(t_{n} ; 0, y\right)\right) \frac{\partial \Psi}{\partial x}\left(t_{n} ; 0, y\right) d y \mid \\
& \leq C\left(\frac{\sigma \Delta x}{\sigma^{2}}\right)=C\left(\frac{\Delta x}{\sigma}\right),
\end{aligned}
$$

where $C$ is a constant independent of $\Delta x$ and $\sigma$. The denominator comes from differentiating the mollifier function in the integrand. Therefore, we get

$$
0 \leq \mathcal{I}_{n}\left(v^{n}\right)_{i}^{2} \leq M_{n}^{2}(1+C(\Delta x / \sigma)) .
$$

Applying this inequality to (24a) leads to

$$
\begin{aligned}
\min _{j} u_{j}^{n+1} & \geq \min _{j}\left[u_{j}^{n}-\Delta t\left(u_{j}^{n}\right)^{2}\right] \\
\max _{j} u_{j}^{n+1} & \left.\leq \max _{j}\left[u_{j}^{n}-\Delta t\left(u_{j}^{n}\right)^{2}\right]+\Delta t M_{n}^{2}+C M_{n}^{2} \Delta t(\Delta x / \sigma)\right) .
\end{aligned}
$$

Consider the function $f(u)=u-\Delta t u^{2}$. If $\Delta t \leq 1 /\left(2 M_{n}\right)$, the function $f$ has a global maximum at $u_{0}=1 /(2 \Delta t) \geq M_{n}$. In the interval $\left[0, M_{n}\right], f(u)$ is strictly increasing, which implies

$$
0 \leq f(u) \leq M_{n}-\Delta t M_{n}^{2}, \quad \text { for all } 0 \leq u \leq M_{n} .
$$

We combine this result with (68) to conclude

$$
0 \leq u_{i}^{n+1} \leq M_{n}+C M_{n}^{2} \Delta t(\Delta x / \sigma), \quad i \in \mathbb{Z} .
$$

Therefore, we have

$$
0 \leq u_{i}^{n+1} \leq M_{n+1}, \quad i \in \mathbb{Z}
$$


with

$$
\left|M_{n+1}-M_{n}\right| \leq C M_{n}^{2} \Delta t(\Delta x / \sigma) \text {. }
$$

Thus, for any finite time $T$, there exists a $\Delta x_{0}>0$ and a constant $M>0$ such that for all $0<\Delta x \leq \Delta x_{0}$, we have $M_{n} \leq M$ and

$$
0 \leq u_{i}^{n}, v_{i}^{n} \leq M, \quad i \in \mathbb{Z}
$$

for $0 \leq n \Delta t \leq T$. $\square$

6.2. Interpolation Error. Given a smooth, bounded function $f(x, t)$, we are interested in the approximation error made by interpolating the discrete values $f\left(\Psi\left(t_{n} ; 0, x_{j}\right), t_{n}\right), j \in \mathbb{Z}$, at $\alpha=\Phi\left(t_{n} ; 0, x_{i}\right)$. We consider the interpolation formula (17) in the case when the characteristics are known exactly. Hence, we approximate

$$
\left.f\left(\alpha, t_{n}\right) \approx \Delta x \sum_{j} \theta_{\sigma}\left(\alpha-\Psi\left(t_{n} ; 0, x_{j}\right)\right) f\left(\Psi\left(t_{n} ; 0, x_{j}\right), t_{n}\right)\right) \frac{\partial \Psi}{\partial x}\left(t_{n} ; 0, x_{j}\right) .
$$

For simplicity, we regard the discrete time $t_{n}$ as a parameter and $f$ as a function of the space variable only. Define $g(x)=\Psi\left(t_{n} ; 0, x\right)$. We have

$$
f(\alpha) \approx \Delta x \sum_{j} \theta_{\sigma}\left(\alpha-g\left(x_{j}\right)\right) f\left(g\left(x_{j}\right)\right) g^{\prime}\left(x_{j}\right) .
$$

Let $y_{j}=g\left(x_{j}\right)$. If $f(\alpha)$ is a smooth function, we can expand $f\left(y_{j}\right)$ around $\alpha$ to obtain

$$
f\left(y_{j}\right)=f(\alpha)+f^{\prime}\left(\eta_{j}\right)\left(y_{j}-\alpha\right)
$$

where $\eta_{j}$ is some point between $y_{j}$ and $\alpha$. The interpolation error is now given by

$$
\begin{aligned}
f(\alpha)-\Delta x \sum_{j} \theta_{\sigma}\left(\alpha-g\left(x_{j}\right)\right) & f\left(g\left(x_{j}\right)\right) g^{\prime}\left(x_{j}\right) \\
=f(\alpha)- & f(\alpha) \Delta x \sum_{j} \theta_{\sigma}\left(\alpha-g\left(x_{j}\right)\right) g^{\prime}\left(x_{j}\right) \\
& -\Delta x \sum_{j} \theta_{\sigma}\left(\alpha-g\left(x_{j}\right)\right) g^{\prime}\left(x_{j}\right) f^{\prime}\left(\eta_{j}\right)\left(g\left(x_{j}\right)-\alpha\right) .
\end{aligned}
$$

The sum over $j$ in the RHS of the above equation includes only the terms for which

$$
\left|g\left(x_{j}\right)-\alpha\right| \leq \sigma .
$$

The number of such terms is of order $O(\sigma / \Delta x)$.

Using the property $\int \theta_{\sigma}(x-y) d y=1$ of the mollifier $\theta_{\sigma}$, we get

$$
\begin{aligned}
\Delta x \sum_{j} \theta_{\sigma}\left(\alpha-g\left(x_{j}\right)\right) g^{\prime}\left(x_{j}\right)= & \Delta x \sum_{j} \theta_{\sigma}\left(\alpha-\Psi\left(t_{n} ; 0, x_{j}\right)\right) \frac{\partial \Psi}{\partial x}\left(t_{n} ; 0, x_{j}\right) \\
& -\int_{\mathbb{R}} \theta_{\sigma}\left(\alpha-\Psi\left(t_{n} ; 0, y\right)\right) \frac{\partial \Psi}{\partial x}\left(t_{n} ; 0, y\right) d y+1 .
\end{aligned}
$$


In the proof of Lemma 4.5 we have shown that

$$
\begin{aligned}
\mid \Delta x \sum_{j} \theta_{\sigma}\left(\alpha-\Psi\left(t_{n} ; 0, x_{j}\right)\right) & \frac{\partial \Psi}{\partial x}\left(t_{n} ; 0, x_{j}\right) \\
& -\int_{\mathbb{R}} \theta_{\sigma}\left(\alpha-\Psi\left(t_{n} ; 0, y\right)\right) \frac{\partial \Psi}{\partial y}\left(t_{n} ; 0, y\right) d y \mid \leq C_{1}\left(\frac{\Delta x}{\sigma}\right),
\end{aligned}
$$

which implies

$$
\left|1-\Delta x \sum_{j} \theta_{\sigma}\left(\alpha-g\left(x_{j}\right)\right) g^{\prime}\left(x_{j}\right)\right| \leq C_{1}\left(\frac{\Delta x}{\sigma}\right) .
$$

We use this estimate in (70) to obtain the following error estimate for the interpolation error:

$$
\begin{aligned}
& \left.\mid f\left(\alpha, t_{n}\right)-\Delta x \sum_{j} \theta_{\sigma}\left(\alpha-\Psi\left(t_{n} ; 0, x_{j}\right)\right) f\left(\Psi\left(t_{n} ; 0, x_{j}\right), t_{n}\right)\right) \frac{\partial \Psi}{\partial x}\left(t_{n} ; 0, x_{j}\right) \mid \\
& \leq C_{1}\left(\frac{\Delta x}{\sigma}\right)+C_{2} \sigma\left\|f^{\prime}\right\|_{L^{\infty}} .
\end{aligned}
$$

REMARK. We remark that higher order approximation can be obtained for sufficiently smooth $f$ by using a shape function satisfying some moment conditions, i.e. $\int x^{k} \theta(x) d x=0$ for $1 \leq k \leq m-1$ for some integer $m>1$. The improved moment error is of order $O\left(\sigma^{m}\right)$ with the error constant depending on the $m$ th order derivative of $f$. However, for the problem we are interested in, $f$ is highly oscillatory. The formal gain of high order moment error could be offset by the large amplitude of the high order derivatives of $f$, leading to an error of the form $O\left((\sigma / \epsilon)^{m}\right)$. Nonetheless, we found from our numerical experiments that high order interpolation methods give more accurate results, especially for the original Broadwell model where there is a resonant interaction among the three components of the solution.

Acknowledgment. The research of T. Hou was supported in part by NSF under the NSF FRG grant DMS-0353838 and ITR Grant ACI-0204932.

\section{REFERENCES}

[1] R.P. Beyer And R.J.Leveque, Analysis of a one-dimensional model for the immersed boundary method, SIAM J. Numer. Anal., 29:2(1992), pp. 332-364.

[2] J. E. BroadwelL, Shock structure in a simple discrete velocity gas, Phys. Fluids, 7(1964), pp. 1243-1247.

[3] T. Carleman, Problèmes mathématiques dans la théorie cinétique de gaz, Publ. Sc. Inst. Mittag-Leffler, Uppsala, 1957.

[4] W. E AND T. Y. Hou, Homogenization and convergence of the vortex method for 2-D Euler equations with oscillatory vorticity fields, Comm. Pure and Appl. Math., 43(1990), pp. $821-855$.

[5] B. Engquist, Computation of oscillatory solutions to partial-differential equations, Lecture Notes in Mathematics, 1270(1987), pp. 10-22. 
[6] B. Engquist and T. Y. Hou, Particle method approximation of oscillatory solutions to hyperbolic differential equations, SIAM J. Numer. Anal., 26:2(1989), pp. 289-319.

[7] B. EngQuist AND J-G. LiU, Numerical methods for oscillatory solutions to hyperbolic problems, Comm. Pure Appl. Math., 46:10(1993), pp. 1327-1361.

[8] T. Y. Hou, Homogenization for semilinear hyperbolic systems with oscillatory data, Comm. Pure and Appl. Math., 41(1988), pp. 471-495.

[9] R. ILLnER, Global existence for two-velocity models of the Boltzmann equation, Math. Methods Appl. Sci., 1(1979), pp. 187-193.

[10] E. Isaacson and H. B. Keller, Analysis of Numerical Methods, Dover Publications, New York, 1994.

[11] D.W. McLaughlin, G. Papanicolaou, and L. Tartar, Weak limits of semilinear hyperbolic systems with oscillating data, volume 230 of Lecture Notes in Phys., pp. 277-289. SpringerVerlag, 1985.

[12] L. TARTAR, Solutions oscillantes des équations de Carleman, Seminaire Goulaouic-MeyerSchwartz, 1980-1981. 